\title{
Targeting SLC3A2 Subunit of System XC- is Essential for m6A Reader YTHDC2 to be an Endogenous Ferroptosis Inducer in Lung Adenocarcinoma
}

\section{Lifang Ma}

Shanghai Chest Hospital: Shanghai Jiao Tong University Affiliated Chest Hospital

\section{Xiao Zhang}

Shanghai Chest Hospital: Shanghai Jiao Tong University Affiliated Chest Hospital

\section{Keke Yu}

Shanghai Chest Hospital: Shanghai Jiao Tong University Affiliated Chest Hospital Xin Xu

Shanghai Chest Hospital: Shanghai Jiao Tong University Affiliated Chest Hospital Tianxiang Chen

Shanghai Chest Hospital: Shanghai Jiao Tong University Affiliated Chest Hospital Yi Shi

Shanghai Jiaotong University: Shanghai Jiao Tong University

\section{Yikun Wang}

Shanghai Chest Hospital: Shanghai Jiao Tong University Affiliated Chest Hospital

\section{Shiyu Qiu}

Shanghai Chest Hospital: Shanghai Jiao Tong University Affiliated Chest Hospital

\section{Susu Guo}

Tongji University Tenth People's Hospital: Shanghai Tenth People's Hospital

\section{Jiangtao Cui}

Shanghai Chest Hospital: Shanghai Jiao Tong University Affiliated Chest Hospital

\section{Yayou Miao}

Shanghai Chest Hospital: Shanghai Jiao Tong University Affiliated Chest Hospital

Xiaoting Tian

Shanghai Chest Hospital: Shanghai Jiao Tong University Affiliated Chest Hospital

\section{Lutao Du}

Second Hospital of Shandong University

\section{Yongchun Yu}

Shanghai Chest Hospital: Shanghai Jiao Tong University Affiliated Chest Hospital Jinjing Xia

Shanghai Chest Hospital: Shanghai Jiao Tong University Affiliated Chest Hospital 
Jiayi Wang ( $\sim$ karajan2@163.com)

Shanghai Jiao Tong University Affiliated Chest Hospital https://orcid.org/0000-0003-1688-2864

\section{Research}

Keywords: Pancreatic ductal adenocarcinoma, cancer-associated-fibroblasts, co-cultures, metastasis, epithelial-mesenchymal transition, cytokines, extracellular vesicles.

Posted Date: January 25th, 2021

DOl: https://doi.org/10.21203/rs.3.rs-152451/v1

License: (c) (1) This work is licensed under a Creative Commons Attribution 4.0 International License. Read Full License 


\section{Abstract}

Background: The $\mathrm{m}^{6} \mathrm{~A}$ reader YT521-B homology containing 2 (YTHDC2) inhibits lung adenocarcinoma (LUAD) tumorigenesis by suppressing solute carrier 7A11 (SLC7A11)-dependent antioxidant function. SLC7A11 is a major functional subunit of system $X_{C}{ }^{-}$. Inhibition of system $X_{C}{ }^{-}$can induce ferroptosis. However, whether suppressing SLC7A11 is sufficient for YTHDC2 to be an endogenous ferroptosis inducer in LUAD is unknown.

Methods: Bioinformatics were used to predict overall survival (OS) and possible correlations. Gene expressions were examined by immunoblotting (IB), reverse transcription-quantitative PCR (RT-qPCR), immunohistochemistry (IHC) and enzyme-linked immunosorbent assay (ELISA). The doxycycline (Dox)inducible YTHDC2 cell model, spontaneous LUAD and xenograft mouse models were used to study the function of YTHDC2. SYTOX green and C11-BODIPY581/591 staining following flow cytometry were used to evaluate cell death and lipid reactive oxygen species (ROS) generation. Luciferase reporter assay, electrophoretic mobility shift assay (EMSA) and chromatin immunoprecipitation (ChIP) were performed to study promoter. RNA immunoprecipitation (RIP), RNA pull down and photoactivatable ribonucleotide crosslinking and immunoprecipitation (PAR-CLIP) were used to determine protein-RNA interactions. Actinomycin D chase experiments were used for RNA stability and four independent LUAD cohorts with a total volume of 384 were recruited to evaluate clinical significance.

Results: Induction of YTHDC2 to a high level can induce ferroptosis in LUAD cells but not in lung and bronchus epithelial cells. In addition to SLC7A11, solute carrier 3A2 (SLC3A2), another subunit of system $\mathrm{X}_{\mathrm{C}}{ }^{-}$was equally important for YTHDC2-induced ferroptosis. YTHDC2 $\mathrm{m}^{6} \mathrm{~A}$-dependently destabilized Homeo box $A 13$ (HOXA13) mRNA because a potential $\mathrm{m}^{6} \mathrm{~A}$ recognition site was identified within its $3^{\prime}$ untranslated region (3'UTR). Interestingly, HOXA13 acted as a transcription factor to stimulate SLC3A2 expression. Thereby, YTHDC2 suppressed SLC3A2 via inhibiting HOXA13 in an $\mathrm{m}^{6} \mathrm{~A}$-indirect manner. Mouse experiments further confirmed the associations among YTHDC2, SLC3A2 and HOXA13, and demonstrated that SLC3A2 and SLC7A11 were both important for YTHDC2-impaired tumor growth and induced lipid peroxidation in vivo. Moreover, higher expression of SLC7A11, SLC3A2 and HOXA13 indicate poorer clinical outcome in YTHDC2-suppressed LUAD patients.

Conclusion: YTHDC2 is a powerful endogenous ferroptosis inducer and targeting SLC3A2 subunit of system $X_{C}{ }^{-}$is essential for this process. Increasing YTHDC2 is an alternative ferroptosis-based therapy to treat LUAD.

\section{Background}

Lung cancer is the most prevalent cancer worldwide, with an approximate 5-year survival rate of $16.6 \%$ [1]. LUAD is the most frequent histological manifestation of lung cancer [2]. Despite the curative methods for treating LUAD have been largely improved in the past few decades [3], the overall survival of LUAD 
patients still far from satisfactory and this might due to the incomprehensive discovery of the specific molecular triggers/targets to improve specific treatments.

Ferroptosis is a newly defined form of regulated cell death resulting from iron-dependent overwhelming lipid peroxidation $[4,5]$. Ferroptosis-based therapy is attractive because many types of cancer cells are more metabolic active and sensitive to ferroptosis as compared to normal cells [6, 7]. However, intracellular antioxidant system can protect cancer cells against ferroptosis [8, 9]. Emerging studies have developed strategies to induce ferroptosis by breaking redox balance in cancer cells. Chemical synthesized small molecules such as erastin and sorafenib can induce ferroptosis via targeting the cystine/glutamate antiporter system $\mathrm{X}_{\mathrm{C}}{ }^{-}$. Such effects impair cystine uptake to block subsequent synthesis of glutathione (GSH), the major intracellular antioxidant and prevent activation of its user, such as peroxidase glutathione peroxidase (GPX4) $[10,11]$. Moreover, the direct inhibition to GPX4 by RASselective lethal 3 (RSL3) can also trigger ferroptosis [12]. Although induction of ferroptosis through small molecules is a promising strategy to treat cancer because of their high efficacy [13,14], some studies also pointed out that these chemicals are highly toxic and hard to metabolize in vivo $[15,16]$. Hence, it is urgent to explore alternative ways to induce ferroptosis at genetic levels. Unfortunately, to the best of our knowledge, the endogenous ferroptosis inducers in LUAD cells are still unknown. However, Prominin2 has been recently identified as a novel endogenous ferroptosis inhibitor to facilitate ferroptosis resistance and prevent cancer cell death [17]. This exciting study hints that we might also have endogenous ferroptosis inducers in our body yet just not been discovered thus far.

N6-methyladenosine $\left(m^{6} A\right)$ is a WER system consisted by the writers $(W)$, erasers $(E)$ and readers $(R)$. Writers are methyltransferases while erasers are enzymes catalyze demethylation $[18,19]$. Unlike writers and erasers, readers belong to the category of RNA binding proteins, recognizing and guiding $\mathrm{m}^{6} \mathrm{~A}$ modified RNAs for subsequent processing $[20,21]$, thereby they are the executors of $m^{6} A$. YTHDC2 is such an $\mathrm{m}^{6} \mathrm{~A}$ reader identified in 2017 [22] and its YTH domain is essential for recognizing and binding with the $\mathrm{m}^{6} \mathrm{~A}$-modified target RNAs $[23,24]$. Dysregulation of YTHDC2 has been linked with tumorigenesis and progression $[25,26]$. Our previous study also demonstrated that YTHDC2 exhibits a tumor suppressor activity to suppress antioxidant function of LUAD cells via accelerating SLC7A11 mRNA decay [24]. SLC7A11 is the major functional subunit of system $\mathrm{X}_{\mathrm{C}}{ }^{-}$. As mentioned above that inhibiting system $\mathrm{X}_{\mathrm{C}}{ }^{-}$ can cause ferroptosis $[10,11]$. However, whether YTHDC2 can act as an endogenous ferroptosis inducer and also whether suppressing SLC7A11 is sufficient for YTHDC2 to trigger system $\mathrm{X}_{C}{ }^{-}$-dependent ferroptosis in LUAD cells still unknown.

SLC7A11 and SLC3A2 are two subunits of system $\mathrm{X}_{\mathrm{C}}{ }^{-}$. Despite SLC7A11 is predominantly important for the function of system $X_{C}{ }^{-}[27,28]$, growing evidences have demonstrated that SLC3A2 is equally important for preventing cells from over lipid peroxidation [29, 30]. Additionally, SLC3A2 is also required for erastin to induce ferroptosis [10]. SLC3A2 acts as a chaperone protein to support the function of SLC7A11 [31, 32]. However, unlike SLC7A11 mRNA, we didn't observe any $\mathrm{m}^{6} \mathrm{~A}$ modification within the SLC3A2 mRNA via methylated RNA immunoprecipitation sequencing (meRIP-seq) from our previous 
study [24], demonstrating that SLC3A2 mRNA might not be directly influenced by YTHDC2 in LUAD cells. SLC3A2 can be transcriptionally regulated under IFN- $\gamma$ stimulation in the intestinal epithelium [33], indicating that YTHDC2 could indirectly regulate SLC3A2 at transcription level. However, whether and how YTHDC2 regulates SLC3A2 and such effects could co-ordinately induce ferroptosis is not clear.

Thereby, we investigated whether the $\mathrm{m}^{6} \mathrm{~A}$ reader $\mathrm{YTHDC} 2$ is an endogenous ferroptosis inducer in LUAD cells. We uncover that induction of YTHDC2 to a high level can induce ferroptosis in LUAD cells. In addition to SLC7A11, another subunit of system $\mathrm{X}_{\mathrm{C}}{ }^{-}$, i.e. SLC3A2 is also required for this process. Transcription factor HOXA13 stimulates SLC3A2 transcription; however, YTHDC2 $\mathrm{m}^{6} \mathrm{~A}$-dependently reduces HOXA13 mRNA stability. The data from the current study supplement our knowledge that YTHDC2 is a powerful endogenous inhibitor of system $\mathrm{X}_{\mathrm{C}}{ }^{-}$because YTHDC2 can not only suppress SLC7A11 directly but also suppress SLC3A2 indirectly. Thereby, increasing YTHDC2 will be an attractive and alternative ferroptosis-based therapy for LUAD in the future.

\section{Materials And Methods}

\section{Clinical specimen and animal experiments}

Clinical samples of paired adjacent and LUAD tissue specimens were the same ones obtained from our previous study [24]. The LUAD patients of cohort\#1, \#2 and \#4 were recruited from Shanghai Chest Hospital of Shanghai Jiao Tong University (Shanghai, China) and the cohort\#3 was recruited from Second Hospital of Shandong University (Jinan, Shandong province, China). Written informed consents were obtained from each enrolled patient according to the guidelines of the Declaration of Helsinki. The $\operatorname{Kras}^{G 12 D}$ and $p 53^{R 172 H}(\mathrm{KP})$-driven spontaneous LUAD mice models with or without overexpressing YTHDC2 were constructed as we previously reported [24]. KPE indicates an expression of empty vector, $\mathrm{KPY}^{\mathrm{WT}}$ indicates an expression of wild type (WT) YTHDC2 and KPE $\triangle \mathrm{YTH}$ indicates an expression of YTHDC2 without the YTH domain. For xenograft experiments, $1.5 \times 10^{7}$ Dox-inducible YTHDC2-expressing H1299 cells were subcutaneously injected into 4-6-week-old athymic nude mice. At day 14 post inoculation, mice were randomly divided into 2 groups for further administrating with or without Dox (30 $\mathrm{mg} / \mathrm{kg}$ ) every other day. Tumors were assessed after sacrificing the mice at day 28 after implantation. The tumor volume was measured as $0.5 \times \mathrm{L} \times \mathrm{W}^{2}$, ( $\mathrm{L}$ indicates tumor length, while $\mathrm{W}$ indicates tumor width). All experiments were approved by institutional ethics committee of Shanghai Chest Hospital.

\section{Monolayer and 3 dimensional (3D) cell culture}

Human embryonic kidney (HEK)-293T, human lung epithelial cell line BEAS-2B, bronchus epithelial cell line 16HBE, human LUAD cell lines NCl-H1299, NCl-H1975 and NCl-H441 were purchased from Cell Bank of the Chinese Academy of Science (Shanghai, China). Possible mycoplasma contamination of all the cell lines was excluded. For monolayer culture, cells were cultured in DMEM (GIBCO, Carlsbad, CA, USA) 
with 10\% FBS (HyClone, Logan, UT, USA) and 1\% penicillin/streptomycin (Invitrogen, Carlsbad, CA, USA). For 3D culture, spheroids were generated in Cultrex ${ }^{\circledR}$ Basement Membrane Extract (BME)-based culture system, which is described previously [24].

\section{Reagents and plasmids}

For the reagents used in this study, Deferoxamine (DFO, $80 \mu \mathrm{M}$, \#D9533), N-acetyl-cysteine (NAC, $1 \mathrm{mM}$, \#A7250), and cycloheximide (CHX, $10 \mu \mathrm{g} / \mathrm{ml}$, \#C7698) were purchased from Sigma (St Louis, MO, USA). 3-Methyladenine (3-MA, $5 \mu \mathrm{M}$, \#HY19312), Chloroquine (25 $\mu \mathrm{M}$, \#HY17589), Bortezomib (100 nM, \#PS341), Actinomycin D (ActD, 5 rg/ml, \#HY17559) were purchased from MedChemExpress (Monmouth, NJ, USA). Ferrostatin-1 (Fer-1, $1 \mu \mathrm{M}$, \#S7243), Necrosulfonamide (0.5 $\mu \mathrm{M}$, \#8251), MG132 (50 $\mu \mathrm{M}$, \#S2619), Dox (\#S4163) and Z-VAD-FMK (20 $\mu \mathrm{M}$, \#S7023) were purchased from Selleck (Houston, TX, USA). GSH (5 mM, \#G8180), DEPC water (\#R1600) and RNasin (\#R8060, $5 \mathrm{U} / \mu \mathrm{L}$ ) were purchased from Solarbio (Beijing, China). C11-BODIPY581/591 (5 $\mu \mathrm{M}$, \#D3861) was purchased from Invitrogen. The YTHDC2, YTHDC2 ${ }^{\triangle Y T H}, Y_{T H D C}{ }^{\text {sg2-resistant (res) }}$ METTL3, METTL3-shRNA (sh), EXOSC10-single-guide (sg) RNA, XRN2-sg, YTHDC2-sg1, YTHDC2-sg2, ATG5-sg and siRNA targeting ATG7 were obtained from our previously studies [24,34]. For crispr/cas9 knockout of SLC7A11 (guide RNA sequence:

GATATCACAGCAGTAGCTGC), SLC3A2 (guide RNA sequence: GGCAGCCGCGGCTAAGTTCA) and HOXA13 (guide RNA sequence: GTACTGTTTGGAACAAGAGG) sgRNAs were cloned into the lentiCrisprV2 plasmids (Addgene, Cambridge, MA, USA). Lentiviral-based plasmids expressing POLR2L, SLC7A11 and SLC3A2 were purchased from Zuorun Biotech Ltd (Shanghai, China). Dox-inducible YTHDC2 and YTHDC2 ${ }^{\triangle T T H}$ were constructed into the lentiviral based pLVX plasmids (BioVision Technology Ltd, Shanghai, China). The primer sequences were listed in Supplementary Table S1.

\section{IB, ELISA, IHC and RT-qPCR}

IB was performed conventionally. The primary antibodies used for IB were: anti-YTHDC2 (Abcam, \#ab176846, \#220160, Cambridge, MA, USA), anti-METTL3 (Abcam, \#ab195352), anti-ATG5 (Abcam, \#ab108327), anti-ATG7 (Cell Signaling Technology (CST), \#8558S, Boston, MA, USA), anti-SLC7A11 (Abcam, \#ab175186), anti-SLC3A2 (Abcam, \#ab108300), anti-EXOSC10 (Abcam, \#ab50558), antiHOXA13 (Abcam, \#ab172570), anti-XRN2 (Abcam, \#ab72181), anti-HA (CST, \#2367S) and anti-GAPDH (Abcam, \#ab181602). The SLC3A2 and HOXA13 protein levels in tissues were also determined by ELISA kits from Lichen Biotech Ltd (Shanghai, China). The tissue microarray assay (TMA) was subjected into IHC using conventional protocols. IHC scores were calculated as we described previously [24]. The antibody used for IHC were: anti-SLC3A2 (Abcam, \#ab108300), anti-HOXA13 (Abcam, \#ab172570) and anti-4-hydroxynonenal (4-HNE) (Abcam, \#ab48506). For RT-qPCR, total RNA was isolated using Trizol reagent (Invitrogen). cDNA was synthesized with RT-PCR Kit (Vazyme Biotech, \#P611, Nanjing, China). The relative mRNA and RNA fragments expression level was quantified by qPCR with a SYBR Kit (Vazyme 
Biotech, \#Q711) and was normalized to the data from GAPDH or IgG. The qPCR primers are listed in Supplementary Table S1.

\section{Luciferase reporter assay}

For the promoter study, truncated versions of the SLC3A2 promoter were cloned into the pGL4-basic plasmids (Promega, Madison, WI, USA). For the analysis of mRNA stability, partial 3' untranslated region (3'UTR) of the HOXA13 mRNA was cloned into the pmir-GLO plasmids (Zuorun Biotech Ltd). For mutation of $3^{\prime}$ UTR, adenosine (A) in the $\mathrm{m}^{6} \mathrm{~A}$ motif was replaced by a cytosine (C). The WT and mutant (Mut) PCR products were synthesised by Generay Biotech Ltd (Shanghai, China). Luciferase activity was detected by the dual luciferase reporter gene assay kit (Promega). The firefly luciferase activities were normalized to the Renilla luciferase activities. The primers used for reporter construction were listed in Supplementary Table S1.

\section{MeRIP-seq, PAR-CLIP, RIP, RNA pull down assay}

MeRIP-seq data were obtained from previous study and PAR-CLIP analysis was measured as previously described [24]. For RIP experiments, cell lysates were incubated with magnetic beads loaded with $5 \mu \mathrm{g}$ antibodies overnight at $4^{\circ} \mathrm{C}$. RNA-protein mixture was then digested by proteinase $\mathrm{K}$. The remaining RNA was finally measured by RT-qPCR with normalization to input. For RNA pull down, probes were synthesized with or without $\mathrm{m}^{6} \mathrm{~A}$ modification at GGAC or mutant GGCC motifs. Briefly, the probes were labelled by biotin (Takara, Dalian, China). Incubated cell lysed with $3 \mu \mathrm{g}$ biotinylated probes at $4^{\circ} \mathrm{C}$ overnight. Then biotin-coupled RNA-protein complex were pulled down using streptavidin magnetic beads (Life Technologies, Carlsbad, CA, USA). After washing, the streptavidin beads were boiled and analyzed for the IB analysis. The primer and probe sequences are supplied in Supplementary Table S1.

\section{EMSA and ChIP}

For EMSA, the WT and mut short oligonucleotide probes were synthesized by GenePharma (Shanghai, China). The probes sequences are listed in Supplementary Table S1. EMSA was performed using the gel shift kit (Promega). For the super shift assay, $0.5 \mu \mathrm{g}, 1 \mu \mathrm{g}$, or $2 \mu \mathrm{g}$ of the anti-HOXA13 (Abcam, \#ab172570) antibody or IgG (CST, \#3900) were added to the nuclear extracts, and incubated at RT for 10 min prior to the DNA binding reaction. All DNA-protein mixtures were resolved in electrophoresis on $5 \%$ native polyacrylamide. For ChIP, it was performed using a ChIP-IT ${ }^{\circledR}$ Express kit (Active Motif, Cat \#53008, Carlsbad, CA, USA) according to the manufacturer's instructions. Protein-DNA complexes were incubated with anti-HOXA13 (Abcam, \# ab172570) or IgG (CST, \#3900) antibodies coupled protein $\mathrm{G}$ beads at $4^{\circ} \mathrm{C}$ overnight. After elution and reverse cross-link, DNA was purified for subsequent qPCR. The probes and primers used for EMSA and ChIP-qPCR were listed in Supplementary Table S1. 


\section{Fluorescence in situ hybridization (FISH)}

Briefly, samples were deparaffinized, digested with proteinase $K$, prehybridized with prehybridization buffer for $1 \mathrm{~h}$ at $37^{\circ} \mathrm{C}$. Slides were incubated with specific Cy3-labeled probes for HOXA13 mRNA overnight. After washing 3 times with saline sodium citrate buffer, slides were incubated with antiYTHDC2 antibody (Invitrogen, \# PA5-67256) and fluorescent secondary antibody (CST, \#4412) for 1h at RT. Finally, nuclei were counterstained with DAPI. All images were collected via a confocal microscope (Leica, wetzlar, German). The probes were listed in Supplementary Table S1.

\section{Cell viability and cell death analysis}

Cell viability was determined using the CellTilter-Glo cell viability assay (Promega, \#G9682) according to manufacturer's instructions. Cell death was measured by staining with SYTOX Green followed by flow cytometry.

\section{Electron microscopic analysis}

To observe the morphological change of mitochondria following induction of YTHDC2, H1299-iYTHDC2 cells were seeded onto 4-well chambered cover glass (Thermo Fisher Scientific, \#155382, Waltham, MA, USA) at a density of 15,000 cells/well and treated with or without Dox for $24 \mathrm{~h}$. Images were captured using the Olympus EM208S transmission electron microscope (TEM, hitachi, Tokyo, Japan).

\section{Measurement of lipid peroxidation}

To detect lipid ROS, the cells were stained with $5 \mu \mathrm{M}$ C11-BODIPY $581 / 591$ for 30 min at $37^{\circ} \mathrm{C}$ followed by microscopic or flow cytometric analysis. To visualize the membrane, cells were incubated with $25 \mu \mathrm{g} / \mathrm{ml}$ Concanavalin A-Alexa Fluor ${ }^{\mathrm{TM}} 350$ (Thermo Scientific, \#C11254) following C11-BODIPY ${ }^{581 / 591}$ staining. Images were taken at emission at 580/600 nm (the non-oxidized form, red) and 490/510 nm (the oxidized form, green). To measure 4-HNE and malondialdehyde (MDA), kits from Abcam (\#238538 and \#118970) were used.

\section{Bioinformatics}

UALCAN was mined to predict the transcriptional expression of SLC3A2. The Kaplan-Meier plotter databases were used to analyze survival information in clinical LUAD patients. The statistics of correlation between SLC3A2 and YTHDC2 were carried out with Starbase database. The HOXA13 binding site within the SLC3A2 promoter was predicted by JASPAR and UCSC database. 


\section{Statistical analysis}

GraphPad Prism 8 software was used for all statistical analyses. Pvalues were calculated with t-test, one-way ANOVA, two-way ANOVA, pearson analysis and the Chi-squared test. Survival curves were generated using the Kaplan-Meier method and compared using the log-rank test. All the values are presented as means \pm SEMs from three independent experiments. The indicated $p$ values $\left({ }^{*} p<0.05\right.$ and $\star * p<0.01)$ were considered statistically significant. N.S. means non-significance.

\section{Results}

\section{YTHDC2 is an endogenous ferroptosis inducer in LUAD cells}

Because it's difficult to maintain LUAD cells at a healthy state when YTHDC2 was constitutively expressed, we thereby established Dox-inducible YTHDC2-expressing lung epithelia cell BEAS2B, bronchus epithelia cell 16HBE and LUAD cells H1299 and H441 to investigate whether YTHDC2 induces ferroptosis (Figure. 1A). Similar to that we reported previously [24], YTHDC2 was downregulated in LUAD cells as compared to lung and bronchus epithelia cells (Figure. 1A). By adding a serial concentration of Dox for 24h, we confirmed that YTHDC2 could be induced dosedependently (Figure. 1A). Under such conditions, cell viability was dose-dependently reduced while cell death and lipid ROS generation was induced in $\mathrm{H} 1299$ and $\mathrm{H} 441$ cells, and such effects could be prevented by simultaneously administrating with Fer-1, a ferroptosis inhibitor (Figure. 1B), suggesting that YTHDC2-induced ferroptosis did occur in LUAD cells. However, BEAS2B and 16HBE showed a resistance to YTHDC2-induced ferroptosis (Figure. 1B). These results demonstrated that YTHDC2-induced ferroptosis might be selectively sensitive to LUAD cells. Meanwhile, we also found that cell proliferation was not affected following induction of YTHDC2 for 24h (Supplementary Figure. 1A). Reasoning that a concentration of 1.6 $\mu \mathrm{M}$ Dox was sufficient to induce a significant YTHDC2 expression and ferroptosis in H1299 and H441 cells (Figure. 1A-B), we didn't increase Dox concentration any more and fixed this concentration to induce YTHDC2 expression for the following study. To investigate the time upon occurrence of ferroptosis following induction of YTHDC2, we monitored H1299 cells and found that YTHDC2 was induced and sustained at a similar high level began at $24 \mathrm{~h}$ following administrating Dox (Figure. 1C). Cell detachment and overt cell death were also observed began at that time (Figure. 1C). Ferroptosis is characterized morphologically by the presence of smaller than normal mitochondria with condensed mitochondrial membrane densities [35, 36], induction of YTHDC2 following treating with $1.6 \mu \mathrm{M}$ Dox for $24 \mathrm{~h}$ caused similar results in $\mathrm{H} 1299$ and $\mathrm{H} 441$ cells (Supplementary Figure. 1B-C). These results demonstrated that ferroptosis could be triggered in LUAD cells once upon the expression of YTHDC2 was induced to a high level.

To exclude other forms of cell death, apoptosis inhibitor Z-VAD-FMK, necrosis inhibitor Necrosulfonamide and autophagy inhibitors 3-MA and Chloroquine were co-treated with Dox. Unlike ferroptosis inhibitors DFO and Fer-1, other cell death inhibitors were unable to prevent YTHDC2-induced cell death (Figure. 1DE), suggesting that at least apoptosis, necrosis and autophagy are not involved. Apoptosis and autophagy were further excluded because YTHDC2-induced cell death was not aggravated when MG132 
and Bortezomib, two proteasome inhibitors can also induce apoptosis, were co-treated (Figure. 1D-E), and induction of YTHDC2 was still able to induce cell death when the expression of ATG5 and ATG7, two key players of autophagy, were impaired (Figure. 1F-G).

The 3D cellular models mimic interactions present in tissues better than conventional monolayer 2D cell culture [37, 38]. We found that induction of YTHDC2 was also effective to induce ferroptosis in 3D spheroids that generated from $\mathrm{H} 1299$ and $\mathrm{H} 441$ cells (Figure. 1H-I). Also, the number and size of the 3D spheroids were significantly suppressed following YTHDC2 induction, by which could be reversed by Fer1 (Figure. $1 \mathrm{H}-\mathrm{I})$, indicating that induction of YTHDC2 might be useful to reduce tumor burden in a ferroptosis-dependent manner.

\section{Discussion}

YTHDC2 was previously reported to inhibit LUAD tumorigenesis by suppressing SLC7A11 mRNA in an $\mathrm{m}^{6} \mathrm{~A}$-dependent manner [24]. However, other potential target transcripts of YTHDC2 have not yet been fully verified. In the current study, we found that in addition to SLC7A11, another subunit of system $\mathrm{X}_{\mathrm{C}}{ }^{-}$, i.e. SLC3A2 is also $\mathrm{m}^{6} \mathrm{~A}$-dependently suppressed by YTHDC2, but rather via an indirect way. Familiar to SLC7A11 mRNA, HOXA13 mRNA can also be destabilized by YTHDC2 because a potential $\mathrm{m}^{6} \mathrm{~A}$ recognition site was identified within the $3^{\prime} U T R$ of HOXA13 mRNA. Thereby, another novel YTHDC2 target transcript is revealed. Interestingly, HOXA13 mRNA encodes HOXA13; a transcription factor has the capacity to stimulate SLC3A2 transcription. Hence, the indirect role of YTHDC2 to suppress SLC3A2 via HOXA13 is also established in this study (Fig. 8).

System $\mathrm{X}_{\mathrm{C}}{ }^{-}$is consisted by SLC7A11 and SLC3A2, due to the dual inhibitory roles of YTHDC2 to suppress both of the two subunits; we believe that YTHDC2 is a powerful endogenous inhibitor to system $\mathrm{X}_{\mathrm{C}}{ }^{-}$in LUAD cells (Fig. 8). Recently, simultaneous transcriptional downregulation of SLC3A2 and SLC7A11 by interferon gamma (IFNY) has been revealed to induce ferroptosis in tumour cells [30]. Although IFNY can efficiently induce ferroptosis in cancer cells, its pharmacological cytotoxicity is still an insurmountable problem $[49,50]$. In comparison to IFNy, we report here that elevating the level of YTHDC2 might be a safer and controllable ferroptosis-based therapy to treat LUAD because even induction of YTHDC2 to a high level didn't confer ferroptosis in lung and bronchus epithelia cells.

To the best of our knowledge, others and we have not reported that SLC3A2 mRNA is $\mathrm{m}^{6} \mathrm{~A}$ methylated. This cannot exclude that the $S L C 3 A 2 \mathrm{mRNA}$ is truly not to be $\mathrm{m}^{6} \mathrm{~A}$ methylated, because at some occasions, failure to identify $\mathrm{m}^{6} \mathrm{~A}$ methylation only indicates that the abundance of certain modification is not high enough to reach the detection threshold [51]. However, such negative results at least manifest that the direct $\mathrm{m}^{6} \mathrm{~A}$ regulation of SLC3A2 is less critical in LUAD cells. As we expected, the indirect $\mathrm{m}^{6} \mathrm{~A}$ modulation of SLC3A2 is essential for YTHDC2 to suppress system $\mathrm{X}_{\mathrm{C}}{ }^{-}$function, and this is mediated by the $\mathrm{m}^{6} \mathrm{~A}$ methylation of the HOXA13 mRNA, which encodes HOXA13, a member of class I homeobox gene family [52]. High expression of HOXA13 is linked with poor survival in cancers [52, 53]. HOXA13 also 
plays pivotal roles in promotion, growth and therapy resistance in a variety of cancers [54-56], suggesting that YTHDC2-mediated suppression of HOXA13 is not only effective for the induction of ferroptosis but also has potential functions to impair tumor growth and reverse therapy resistance. Interestingly, overexpression of HOXA13 confers Sorafenib resistance in hepatocellular carcinoma [52], and Sorafenib itself has been well established as an inhibitor to suppress system $X_{C}{ }^{-}[10,11]$. It's not difficult to understand why this happens because HOXA13 has the potential to stimulate system $\mathrm{X}_{\mathrm{C}}{ }^{-}$ function via upregulating SLC3A2 expression. Given that $\mathrm{HOXA13}$ and system $\mathrm{X}_{\mathrm{C}}{ }^{-}$are closely associated, the $\mathrm{m}^{6} \mathrm{~A}$-dependent indirect inhibition to SLC3A2 via suppressing HOXA13 cannot be ignored for YTHDC2 to act as an endogenous ferroptosis inducer.

YTHDC2 has been previous showed to promote translation of target mRNA by recognizing $m^{6} A$ residues located within the protein-coding region [57]. Our present and previous studies [24] provide another two examples, i.e. HOXA13 and SLC7A11 mRNAs to expand our understanding that YTHDC2 also recognizes $\mathrm{m}^{6} \mathrm{~A}$ modifications within the $3^{\prime}$ UTR regions. Reasoning that the $\mathrm{m}^{6} \mathrm{~A}$ modifications are highly enriched near the stop codons or within the $3^{\prime} \mathrm{UTR}$ of the target transcripts [58], YTHDC2 recognition of $\mathrm{m}^{6} \mathrm{~A}$ modification within $3^{\prime} U T R$ might be more important. The fates of the target transcripts, to a certain extent are determined by the location of $\mathrm{m}^{6} \mathrm{~A}$ sites [59]. It has been well accepted that the $3^{\prime} \mathrm{UTR}$ is essential for the stability of variety of mRNAs in eukaryotes because it's also the target site for regulatory molecules, such as microRNAs, or contains regulatory motifs, such as AU-rich elements $[60,61]$. This also supports that recognizing $\mathrm{m}^{6} \mathrm{~A}$ sites located within the $3^{\prime} \mathrm{UTR}$ might be critical for YTHDC2 to reduce mRNA stability. The mRNA degradation often begins with the removal of the poly(A) tail at the end of $3^{\prime} U T R$, a process named as deadenylation [62]. After this, the mRNAs can be rapidly degraded with RNA exosome [63]. EXOSC10, a catalytic subunit of the RNA exosome, is the major exonuclease responsible for 3'-5' exonucleolytic activity $[45,64]$. Of note, we found that EXOSC10 is also required for YTHDC2 to destabilize both SLC7A11 and HOXA13 mRNAs. Thereby, the model underlying how YTHDC2 facilitates degradation of target mRNAs following $\mathrm{m}^{6} \mathrm{~A}$ methylation might be established; however, how YTHDC2 mediates deadenylation of target transcripts to initiate RNA degradation and how RNA exosome works afterwards are need to be answered in our future work.

\section{Conclusion}

The $\mathrm{m}^{6} \mathrm{~A}$ reader YTHDC2 is identified as an endogenous ferroptosis inducer via targeting system $\mathrm{X}_{\mathrm{C}}{ }^{-}$in LUAD cells. In addition to the direct suppression of SLC7A11, the indirect $\mathrm{m}^{6} \mathrm{~A}$-dependent suppression of SLC3A2 via HOXA13 expands our knowledge that both two subunits of system $\mathrm{X}_{\mathrm{C}}{ }^{-}$are dispensable for YTHDC2 to induce ferroptosis. Increasing YTHDC2 might be an alternative strategy to supplement small chemical molecules-based ferroptosis therapy to improve treatment of LUAD in the future.

\section{Abbreviations}




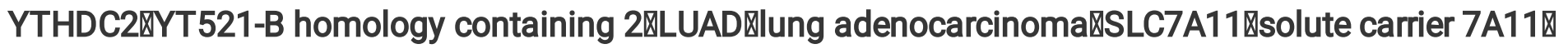

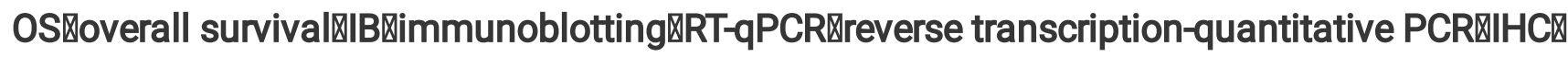

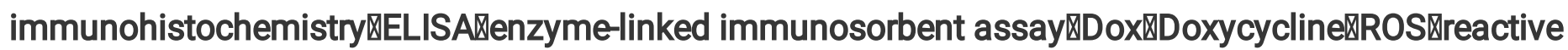

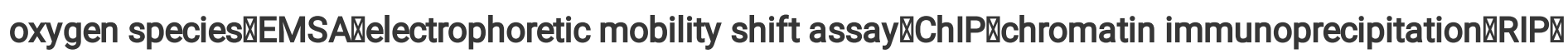
RNA immunoprecipitationखPAR-CLIP区photoactivatable ribonucleotide crosslinking and

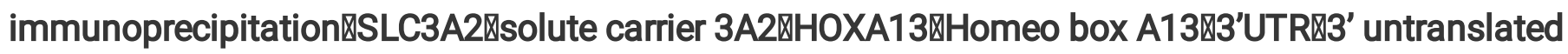

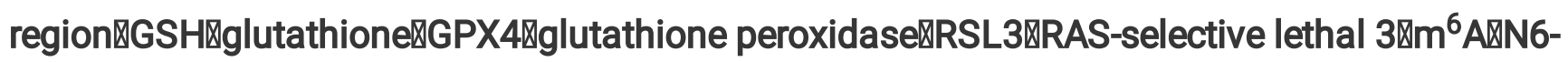

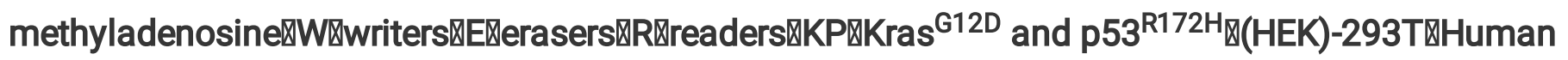
embryonic kidney『BMEखCultrex Basement Membrane ExtractðDFOهDeferoxamine区NAC『N-acetyl-

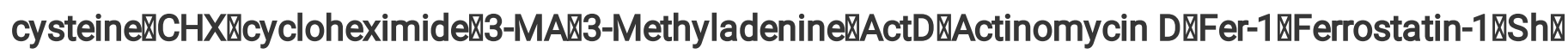

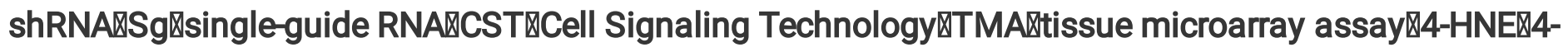

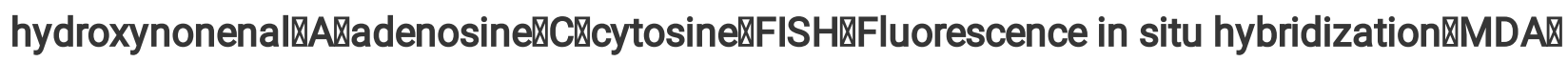

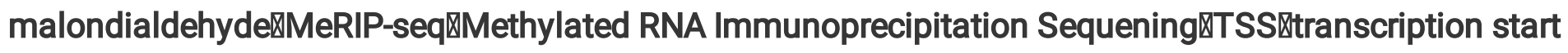
site区TF囚transcription factor, RNA polymerase II (poly II).

\section{Declarations}

Acknowledgements

None.

Author's contributions

L.M., X.Z. and K.Y. researched, analyzed data and analyzed clinical samples. T. C., X.X. and Y.S. collected and analyzed clinical samples. Y.W., S.Q., S.G., J. C., Y.M., X.T. and L.D. researched and analyzed data. Y. Y and J.X. contributed to the discussion. Y.Y. and J. W. designed the study and wrote the manuscript.

\section{Funding}

This work was supported by the National Natural Science Foundation of China $(81871907,81822029$, 81672332, 81902869, 81902315), Shanghai Municipal Education Commission-Gaofeng Clinical Medicine (20191834), Shanghai Rising Star Program (18QA1403400), Shanghai Municipal Commission of Health and Family Planning (2017YQ024), Grant Support "Chen Guang" project supported by Shanghai Municipal Education Commission and Shanghai Education Development Foundation (18CG16), Shanghai Sailing Program (19YF1444800), and Nurture projects for basic research of Shanghai Chest Hospital (2018YNJCQ06, 2018YNJCM01).

Availability of data and materials

The data sets used and/or analyzed during the current study are available from the corresponding author on reasonable request.

Ethics approval and consent to participate 
Our study draft was approved by the institutional review boards of Shanghai Chest Hospital and the second Hospital of Shandong University and written informed consents have been received from each patient.

Consent for publication

Written informed consent for publication was obtained from all the participants.

Competing interests

The authors declared that no competing of interests existing in this study.

\section{References}

1. Bray F, Ferlay J, Soerjomataram I, Siegel RL, Torre LA, Jemal A. Global cancer statistics 2018: GLOBOCAN estimates of incidence and mortality worldwide for 36 cancers in 185 countries. CA Cancer J Clin. 2018;68:394-424.

2. Lee JJ, Park S, Park H, Kim S, Lee J, Lee J, Youk J, Yi K, An Y, Park IK, et al. Tracing Oncogene Rearrangements in the Mutational History of Lung Adenocarcinoma. Cell. 2019;177:1842-57 e1821.

3. Xu JY, Zhang C, Wang X, Zhai L, Ma Y, Mao Y, Qian K, Sun C, Liu Z, Jiang S, et al. Integrative Proteomic Characterization of Human Lung Adenocarcinoma. Cell. 2020;182:245-61 e217.

4. Dixon SJ, Lemberg KM, Lamprecht MR, Skouta R, Zaitsev EM, Gleason CE, Patel DN, Bauer AJ, Cantley AM, Yang WS, et al. Ferroptosis: an iron-dependent form of nonapoptotic cell death. Cell. 2012;149:1060-72.

5. Cao JY, Dixon SJ. Mechanisms of ferroptosis. Cell Mol Life Sci. 2016;73:2195-209.

6. Wu J, Minikes AM, Gao M, Bian H, Li Y, Stockwell BR, Chen ZN, Jiang X. Intercellular interaction dictates cancer cell ferroptosis via NF2-YAP signalling. Nature. 2019;572:402-6.

7. Lee JY, Nam M, Son HY, Hyun K, Jang SY, Kim JW, Kim MW, Jung Y, Jang E, Yoon SJ, et al. Polyunsaturated fatty acid biosynthesis pathway determines ferroptosis sensitivity in gastric cancer. Proc Natl Acad Sci U S A. 2020;117:32433-42.

8. Doll S, Freitas FP, Shah R, Aldrovandi M, da Silva MC, Ingold I, Goya Grocin A, Xavier da Silva TN, Panzilius $\mathrm{E}$, Scheel $\mathrm{CH}$, et al. FSP1 is a glutathione-independent ferroptosis suppressor. Nature. 2019;575:693-8.

9. Tang H, Li C, Zhang Y, Zheng H, Cheng Y, Zhu J, Chen X, Zhu Z, Piao JG, Li F. Targeted Manganese doped silica nano GSH-cleaner for treatment of Liver Cancer by destroying the intracellular redox homeostasis. Theranostics. 2020;10:9865-87.

10. Dixon SJ, Patel DN, Welsch M, Skouta R, Lee ED, Hayano M, Thomas AG, Gleason CE, Tatonetti NP, Slusher BS, Stockwell BR. Pharmacological inhibition of cystine-glutamate exchange induces endoplasmic reticulum stress and ferroptosis. Elife. 2014;3:e02523. 
11. Song X, Zhu S, Chen P, Hou W, Wen Q, Liu J, Xie Y, Liu J, Klionsky DJ, Kroemer G, et al. AMPKMediated BECN1 Phosphorylation Promotes Ferroptosis by Directly Blocking System Xc(-) Activity. Curr Biol. 2018;28:2388-99 e2385.

12. Yang WS, SriRamaratnam R, Welsch ME, Shimada K, Skouta R, Viswanathan VS, Cheah JH, Clemons PA, Shamji AF, Clish CB, et al. Regulation of ferroptotic cancer cell death by GPX4. Cell. 2014;156:317-31.

13. Conrad M, Pratt DA. The chemical basis of ferroptosis. Nat Chem Biol. 2019;15:1137-47.

14. Zou Y, Palte MJ, Deik AA, Li H, Eaton JK, Wang W, Tseng YY, Deasy R, Kost-Alimova M, Dancik V, et al. A GPX4-dependent cancer cell state underlies the clear-cell morphology and confers sensitivity to ferroptosis. Nat Commun. 2019;10:1617.

15. Sun X, Ou Z, Chen R, Niu X, Chen D, Kang R, Tang D. Activation of the p62-Keap1-NRF2 pathway protects against ferroptosis in hepatocellular carcinoma cells. Hepatology. 2016;63:173-84.

16. Ji X, Qian J, Rahman SMJ, Siska PJ, Zou Y, Harris BK, Hoeksema MD, Trenary IA, Heidi C, Eisenberg R, et al. XCT (SLC7A11)-mediated metabolic reprogramming promotes non-small cell lung cancer progression. Oncogene. 2018;37:5007-19.

17. Belavgeni A, Bornstein SR, Linkermann A. Prominin-2 Suppresses Ferroptosis Sensitivity. Dev Cell. 2019;51:548-9.

18. Fu Y, Dominissini D, Rechavi G, He C. Gene expression regulation mediated through reversible $\mathrm{m}(6) A$ RNA methylation. Nat Rev Genet. 2014;15:293-306.

19. Lan Q, Liu PY, Haase J, Bell JL, Huttelmaier S, Liu T. The Critical Role of RNA m(6)A Methylation in Cancer. Cancer Res. 2019;79:1285-92.

20. Seo KW, Kleiner RE. YTHDF2 Recognition of N(1)-Methyladenosine (m(1)A)-Modified RNA Is Associated with Transcript Destabilization. ACS Chem Biol. 2020;15:132-9.

21. Shi $\mathrm{H}$, Wei J, He C. Where, When, and How: Context-Dependent Functions of RNA Methylation Writers, Readers, and Erasers. Mol Cell. 2019;74:640-50.

22. Hsu PJ, Zhu Y, Ma H, Guo Y, Shi X, Liu Y, Qi M, Lu Z, Shi H, Wang J, et al. Ythdc2 is an N(6)methyladenosine binding protein that regulates mammalian spermatogenesis. Cell Res. 2017;27:1115-27.

23. Kretschmer J, Rao $H$, Hackert $P$, Sloan $K E$, Hobartner $C$, Bohnsack MT: The $m(6) A$ reader protein YTHDC2 interacts with the small ribosomal subunit and the 5'-3' exoribonuclease XRN1. RNA 2018, 24:1339-1350.

24. Ma L, Chen T, Zhang X, Miao Y, Tian X, Yu K, Xu X, Niu Y, Guo S, Zhang C, et al. The m(6)A reader YTHDC2 inhibits lung adenocarcinoma tumorigenesis by suppressing SLC7A11-dependent antioxidant function. Redox Biol. 2021;38:101801.

25. Li Y, Zheng JN, Wang EH, Gong CJ, Lan KF, Ding X. The m6A reader protein YTHDC2 is a potential biomarker and associated with immune infiltration in head and neck squamous cell carcinoma. PeerJ. 2020;8:e10385. 
26. Sun $S$, Han $Q$, Liang M, Zhang $Q$, Zhang J, Cao J. Downregulation of $m(6)$ A reader YTHDC2 promotes tumor progression and predicts poor prognosis in non-small cell lung cancer. Thorac Cancer. 2020;11:3269-79.

27. Liu J, Xia X, Huang P. xCT: A Critical Molecule That Links Cancer Metabolism to Redox Signaling. Mol Ther. 2020;28:2358-66.

28. Yamaguchi I, Yoshimura SH, Katoh H. High cell density increases glioblastoma cell viability under glucose deprivation via degradation of the cystine/glutamate transporter XCT (SLC7A11). J Biol Chem. 2020;295:6936-45.

29. Zhang L, Liu W, Liu F, Wang Q, Song M, Yu Q, Tang K, Teng T, Wu D, Wang X, et al: IMCA Induces Ferroptosis Mediated by SLC7A11 through the AMPK/mTOR Pathway in Colorectal Cancer. Oxid Med Cell Longev 2020, 2020:1675613.

30. Wang W, Green M, Choi JE, Gijon M, Kennedy PD, Johnson JK, Liao P, Lang X, Kryczek I, Sell A, et al. CD8(+) T cells regulate tumour ferroptosis during cancer immunotherapy. Nature. 2019;569:270-4.

31. Nakamura E, Sato M, Yang H, Miyagawa F, Harasaki M, Tomita K, Matsuoka S, Noma A, Iwai K, Minato N. 4F2 (CD98) heavy chain is associated covalently with an amino acid transporter and controls intracellular trafficking and membrane topology of 4F2 heterodimer. J Biol Chem. 1999;274:3009-16.

32. Koppula P, Zhang Y, Zhuang L, Gan B. Amino acid transporter SLC7A11/xCT at the crossroads of regulating redox homeostasis and nutrient dependency of cancer. Cancer Commun (Lond). 2018;38:12.

33. Yan Y, Dalmasso G, Sitaraman S, Merlin D. Characterization of the human intestinal CD98 promoter and its regulation by interferon-gamma. Am J Physiol Gastrointest Liver Physiol. 2007;292:G535-45.

34. Guo S, Chen Y, Yang Y, Zhang X, Ma L, Xue X, Qiao Y, Wang J. TRIB2 modulates proteasome function to reduce ubiquitin stability and protect liver cancer cells against oxidative stress. Cell Death Dis. 2021;12:42.

35. Chen Y, Liu S, Li J, Li Z, Quan J, Liu X, Tang Y, Liu B: The Latest View on the Mechanism of Ferroptosis and Its Research Progress in Spinal Cord Injury. Oxid Med Cell Longev 2020, 2020:6375938.

36. Du J, Zhou Y, Li Y, Xia J, Chen Y, Chen S, Wang X, Sun W, Wang T, Ren X, et al. Identification of Frataxin as a regulator of ferroptosis. Redox Biol. 2020;32:101483.

37. Klimkiewicz K, Weglarczyk K, Collet G, Paprocka M, Guichard A, Sarna M, Jozkowicz A, Dulak J, Sarna T, Grillon C, Kieda C. A 3D model of tumour angiogenic microenvironment to monitor hypoxia effects on cell interactions and cancer stem cell selection. Cancer Lett. 2017;396:10-20.

38. Xu R, Zhou X, Wang S, Trinkle C. Tumor organoid models in precision medicine and investigating cancer-stromal interactions. Pharmacol Ther. 2021;218:107668.

39. Huang Y, Dai Z, Barbacioru C, Sadee W. Cystineglutamate transporter SLC7A11 in cancer chemosensitivity and chemoresistance. Cancer Res. 2005;65:7446-54. 
40. Yang Y, Yee D. IGF-I regulates redox status in breast cancer cells by activating the amino acid transport molecule XC. Cancer Res. 2014;74:2295-305.

41. Doll S, Proneth B, Tyurina YY, Panzilius E, Kobayashi S, Ingold I, Irmler M, Beckers J, Aichler M, Walch A, et al. ACSL4 dictates ferroptosis sensitivity by shaping cellular lipid composition. Nat Chem Biol. 2017;13:91-8.

42. Habib E, Linher-Melville K, Lin HX, Singh G. Expression of XCT and activity of system Xc(-) are regulated by NRF2 in human breast cancer cells in response to oxidative stress. Redox Biol. 2015;5:33-42.

43. Yang C, Ponticelli AS. Evidence that RNA polymerase II and not TFIIB is responsible for the difference in transcription initiation patterns between Saccharomyces cerevisiae and Schizosaccharomyces pombe. Nucleic Acids Res. 2012;40:6495-507.

44. Makino DL, Schuch B, Stegmann E, Baumgartner M, Basquin C, Conti E. RNA degradation paths in a 12-subunit nuclear exosome complex. Nature. 2015;524:54-8.

45. Memet I, Doebele C, Sloan KE, Bohnsack MT. The G-patch protein NF-kappaB-repressing factor mediates the recruitment of the exonuclease XRN2 and activation of the RNA helicase DHX15 in human ribosome biogenesis. Nucleic Acids Res. 2017;45:5359-74.

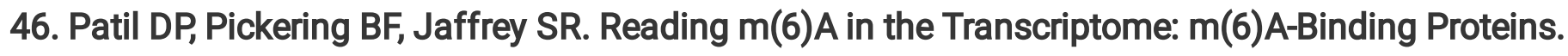
Trends Cell Biol. 2018;28:113-27.

47. Zhang C, Huang S, Zhuang H, Ruan S, Zhou Z, Huang K, Ji F, Ma Z, Hou B, He X. YTHDF2 promotes the liver cancer stem cell phenotype and cancer metastasis by regulating OCT4 expression via m6A RNA methylation. Oncogene. 2020;39:4507-18.

48. Lin X, Chai G, Wu Y, Li J, Chen F, Liu J, Luo G, Tauler J, Du J, Lin S, et al: RNA m(6)A methylation regulates the epithelial mesenchymal transition of cancer cells and translation of Snail. Nat Commun 2019, 10:2065.

49. Fuji N, Ueda Y, Fujiwara H, Toh T, Yoshimura T, Yamagishi H. Antitumor effect of alphagalactosylceramide (KRN7000) on spontaneous hepatic metastases requires endogenous interleukin 12 in the liver. Clin Cancer Res. 2000;6:3380-7.

50. Ding G, Shen T, Yan C, Zhang M, Wu Z, Cao L. IFN-gamma down-regulates the PD-1 expression and assist nivolumab in PD-1-blockade effect on CD8 + T-lymphocytes in pancreatic cancer. BMC Cancer. 2019;19:1053.

51. Wang Y, Zhang Z, Sepich-Poore C, Zhang L, Xiao Y, He C. LEAD-m(6) A-seq for Locus-Specific Detection of N(6) -Methyladenosine and Quantification of Differential Methylation. Angew Chem Int Ed Engl 2020.

52. Quagliata L, Quintavalle C, Lanzafame M, Matter MS, Novello C, di Tommaso L, Pressiani T, Rimassa L, Tornillo L, Roncalli M, et al. High expression of HOXA13 correlates with poorly differentiated hepatocellular carcinomas and modulates sorafenib response in in vitro models. Lab Invest. 2018;98:95-105. 
53. Shi Q, Shen L, Dong B, Fu H, Kang X, Dai L, Yang Y, Yan W, Chen KN. Downregulation of HOXA13 sensitizes human esophageal squamous cell carcinoma to chemotherapy. Thorac Cancer. 2018;9:836-46.

54. Wu DC, Wang SSW, Liu CJ, Wuputra K, Kato K, Lee YL, Lin YC, Tsai MH, Ku CC, Lin WH, et al. Reprogramming Antagonizes the Oncogenicity of HOXA13-Long Noncoding RNA HOTTIP Axis in Gastric Cancer Cells. Stem Cells. 2017;35:2115-28.

55. Gu ZD, Shen LY, Wang H, Chen XM, Li Y, Ning T, Chen KN. HOXA13 promotes cancer cell growth and predicts poor survival of patients with esophageal squamous cell carcinoma. Cancer Res. 2009;69:4969-73.

56. Hoss AG, Labadorf A, Latourelle JC, Kartha VK, Hadzi TC, Gusella JF, MacDonald ME, Chen JF, Akbarian S, Weng Z, et al. miR-10b-5p expression in Huntington's disease brain relates to age of onset and the extent of striatal involvement. BMC Med Genomics. 2015;8:10.

57. Mao Y, Dong L, Liu XM, Guo J, Ma H, Shen B, Qian SB. m(6)A in mRNA coding regions promotes translation via the RNA helicase-containing YTHDC2. Nat Commun. 2019;10:5332.

58. Dominissini D, Moshitch-Moshkovitz S, Schwartz S, Salmon-Divon M, Ungar L, Osenberg S, Cesarkas $\mathrm{K}$, Jacob-Hirsch J, Amariglio N, Kupiec M, et al. Topology of the human and mouse m6A RNA methylomes revealed by m6A-sEq. Nature. 2012;485:201-6.

59. Boulias K, Toczydlowska-Socha D, Hawley BR, Liberman N, Takashima K, Zaccara S, Guez T, Vasseur JJ, Debart F, Aravind L, et al. Identification of the m(6)Am Methyltransferase PCIF1 Reveals the Location and Functions of m(6)Am in the Transcriptome. Mol Cell. 2019;75:631-43 e638.

60. Moraes KC, Wilusz CJ, Wilusz J. CUG-BP binds to RNA substrates and recruits PARN deadenylase. RNA. 2006;12:1084-91.

61. Giraldez AJ, Mishima Y, Rihel J, Grocock RJ, Van Dongen S, Inoue K, Enright AJ, Schier AF. Zebrafish MiR-430 promotes deadenylation and clearance of maternal mRNAs. Science. 2006;312:75-9.

62. Tucker M, Valencia-Sanchez MA, Staples RR, Chen J, Denis CL, Parker R. The transcription factor associated Ccr4 and Caf1 proteins are components of the major cytoplasmic mRNA deadenylase in Saccharomyces cerevisiae. Cell. 2001;104:377-86.

63. Anderson JS, Parker RP. The $3^{\prime}$ to $5^{\prime}$ degradation of yeast mRNAs is a general mechanism for mRNA turnover that requires the SKI2 DEVH box protein and $3^{\prime}$ to $5^{\prime}$ exonucleases of the exosome complex. EMBO J. 1998;17:1497-506.

64. Domingo-Prim J, Endara-Coll M, Bonath F, Jimeno S, Prados-Carvajal R, Friedlander MR, Huertas $P$, Visa N. EXOSC10 is required for RPA assembly and controlled DNA end resection at DNA doublestrand breaks. Nat Commun. 2019;10:2135.

\section{Figures}


A

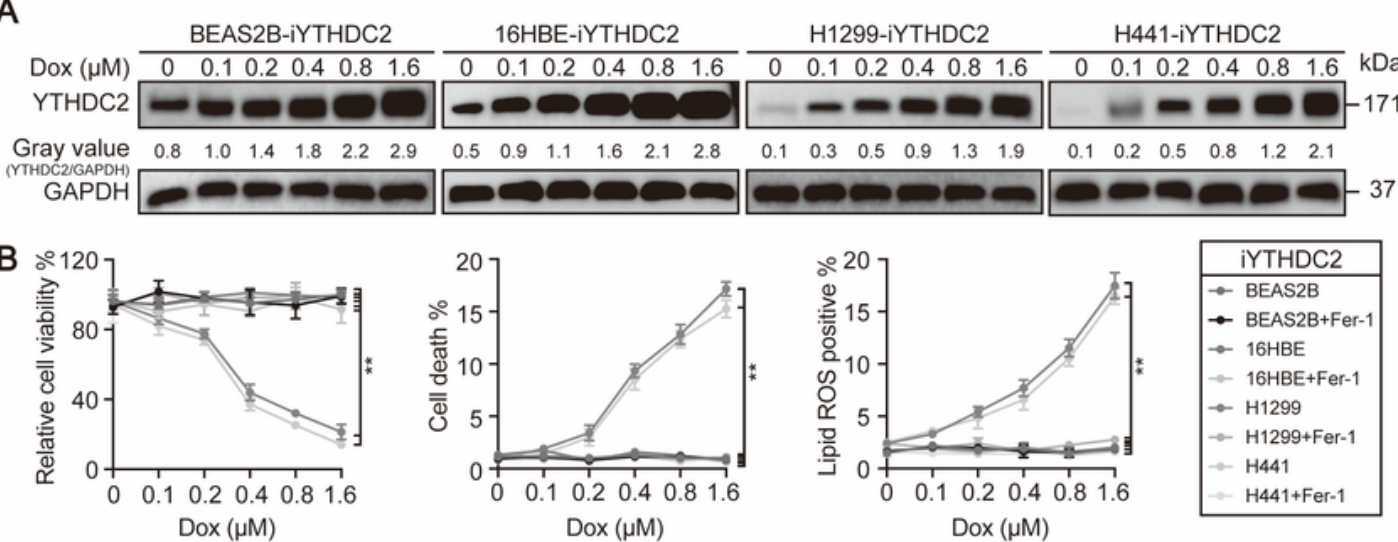

C
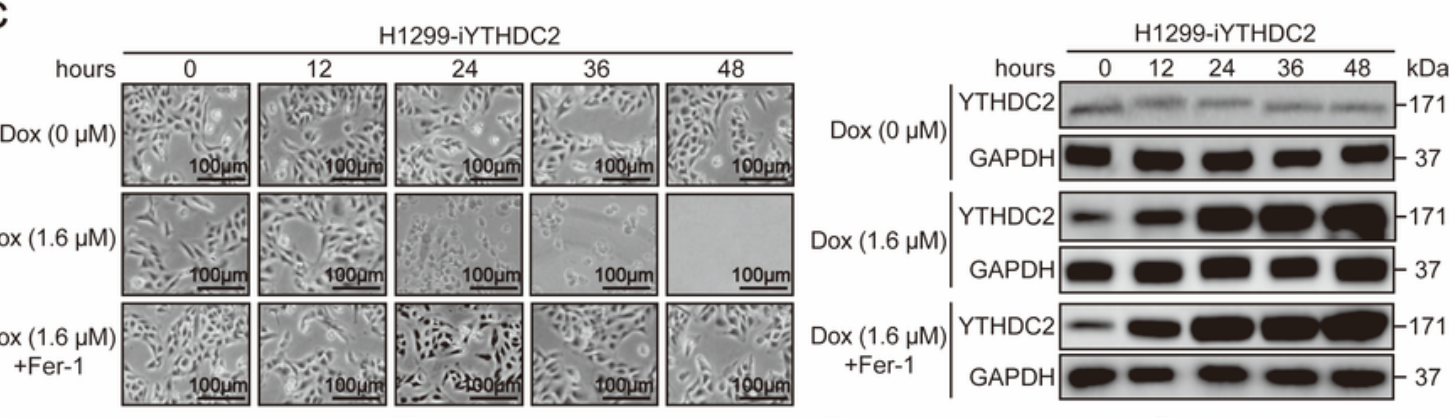

D

$\mathrm{E}$

$\mathrm{H}$
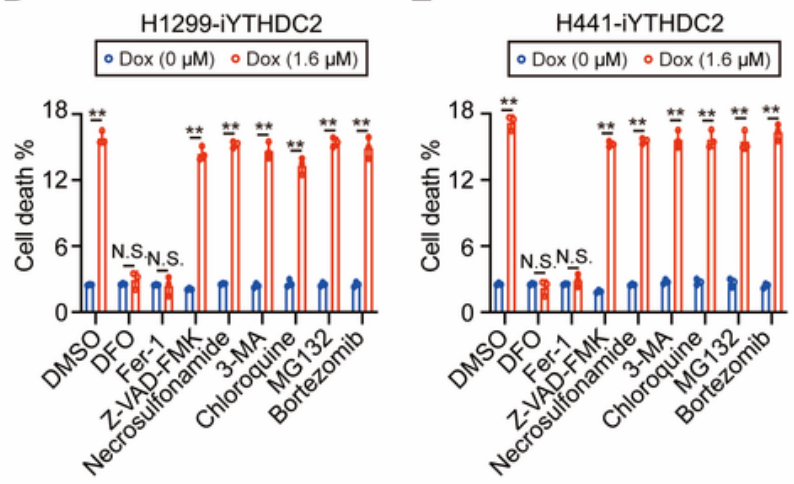

$\mathrm{F}$

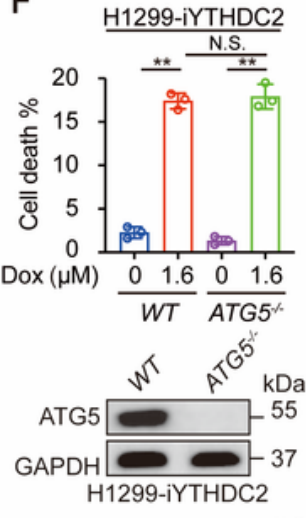

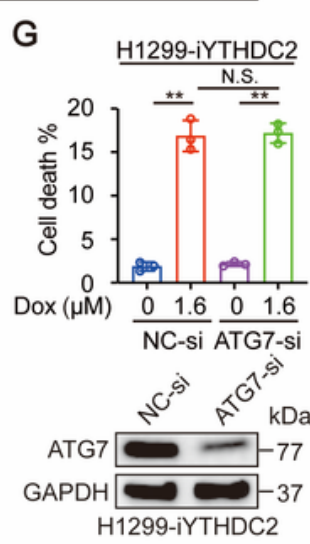

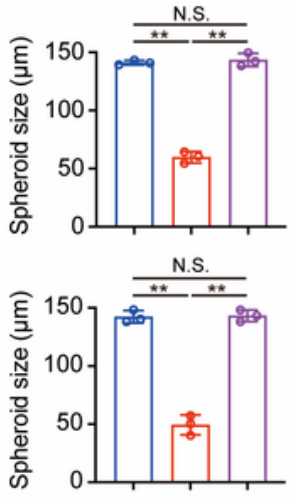

$\square \operatorname{Dox}(0 \mu \mathrm{M})$

$\square$ Dox $(1.6 \mu \mathrm{M})+$ Fer-1

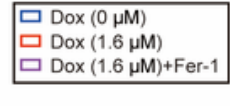

I
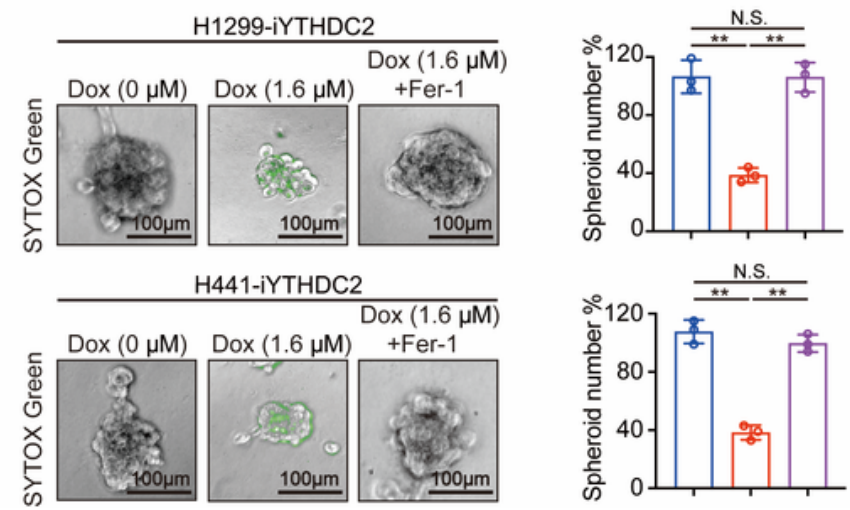

Figure 1

YTHDC2 is an endogenous ferroptosis inducer. (A) Induction of YTHDC2 by indicated concentration of Dox for 24h. (B) Ferroptosis was sensitive in LUAD cells. Cell viability, cell death and lipid ROS generation were measured by ATP-based assay, staining with SYTOX Green and C11-BODIPY581/591 following flow cytometry after treating with indicated concentration of Dox, with or without Fer-1 (1 $\mu \mathrm{M})$ for $24 \mathrm{~h}$. (C) Changes of cell morphology and YTHDC2 expression following treating with indicated concentration of 
Dox in the absence or presence of Fer-1 $(1 \mu \mathrm{M})$ for indicated hours. Scale bar, $100 \mu \mathrm{m}$. (D-E) Cell death was measured by staining with SYTOX Green following flow cytometry in $\mathrm{H} 1299$ (D) and H411 cells (E) treating with indicated concentration of Dox in the absence or presence of DFO $(80 \mu \mathrm{M})$, Fer-1 (1 $\mu \mathrm{M})$, ZVAD-FMK $(20 \mu \mathrm{M})$, Necrosulfonamide $(0.5 \mu \mathrm{M}), 3-\mathrm{MA}(5 \mu \mathrm{M})$, Chloroquine $(25 \mu \mathrm{M}), \mathrm{MG} 132(50 \mu \mathrm{M})$ or Bortezomib (100 nM) for 24h. (F-G) Cell death was measured in H1299 cells with or without KO of ATG5 (F) and knock down of ATG7 (G) following treating with or without Dox for 24h. (H-l) 3D-clutured

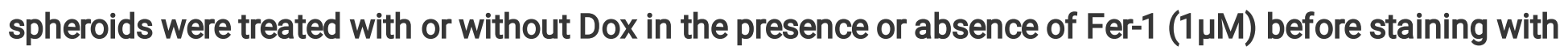
SYTOX Green. The amounts and sizes of spheroids were measured for those with $\Phi$ values of greater than $30 \mu \mathrm{m}$, but smaller than $150 \mu \mathrm{m}$. Scale bar, $100 \mu \mathrm{m}$. Data are presented as means \pm SEMs from three independent experiments. Statistical analysis was performed using two-way ANOVA (B), $t$ test (D, E) and one-way ANOVA (F-I). **p $<0.01$ and N.S., non-significance. 
A

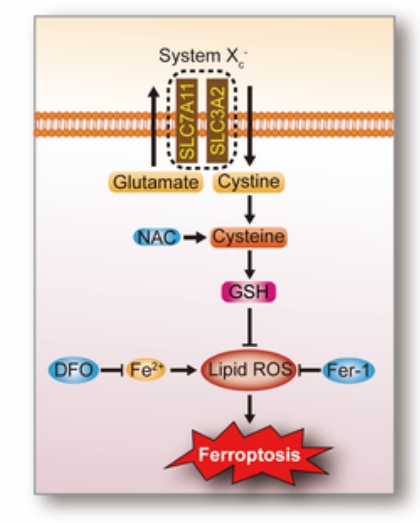

F

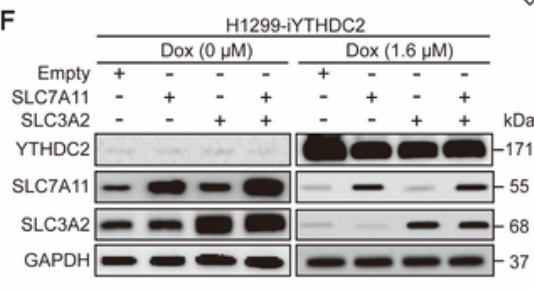

$\mathrm{H}$
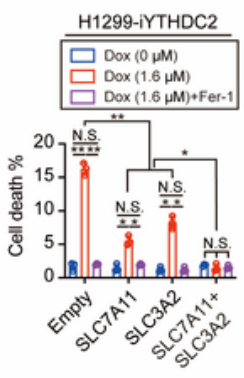

L
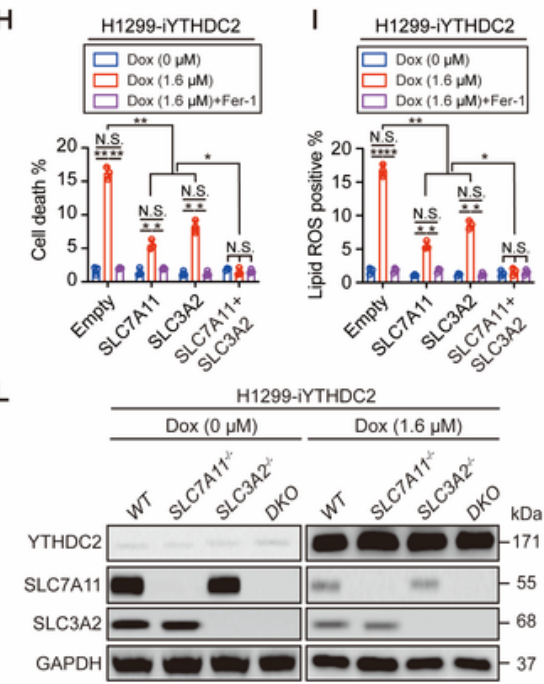

N

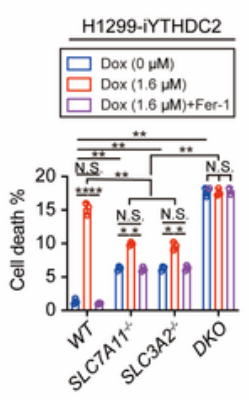

0
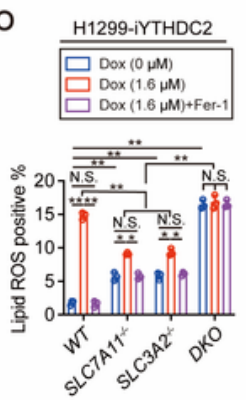

D
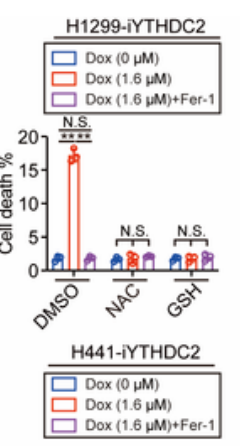

E

c

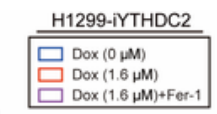

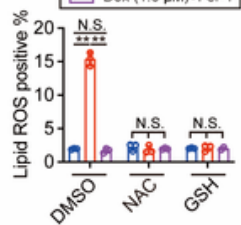

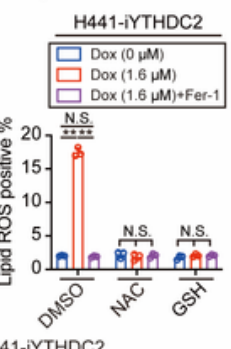

G
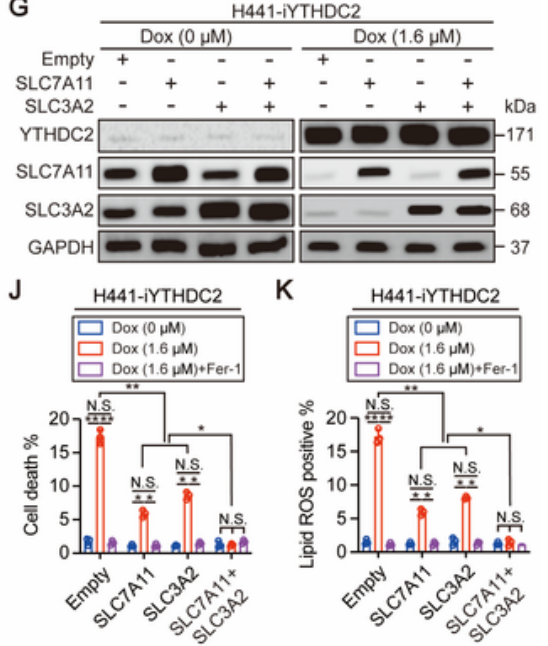

M

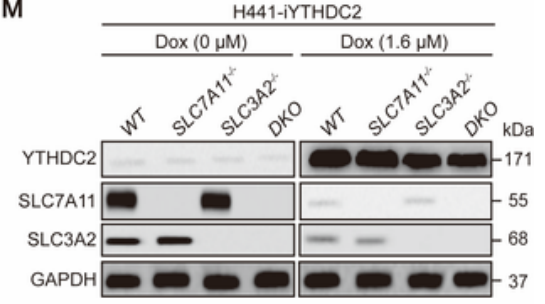

P
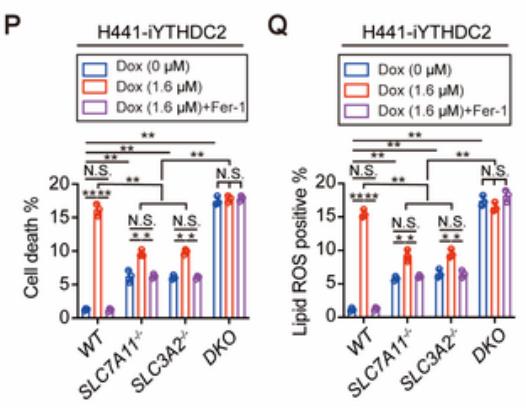

Figure 2

YTHDC2-induced ferroptosis is via targeting SLC7A11 and SLC3A2 (A) Illustration of the system XCdependent metabolism and its role in ferroptotsis. (B-E) Cell death (B, D) and lipid ROS generation (C, E) were measured in $\mathrm{H} 1299$ (B-C) and H441 cells (D-E) following treating with indicated concentration of Dox in the absence or presence of Fer-1 $(1 \mu \mathrm{M})$, NAC (1 mM) or GSH (5 mM) for $24 \mathrm{~h}$. (F-G) SLC7A11 and SLC3A2 were reconstituted in H1299 (F) and H441 cells (G) treating with indicated concentration of Dox 
for $24 \mathrm{~h}$, as measured by IB. (H-K) Cell death and lipid ROS generation were measured in H1299 $(\mathrm{H}-\mathrm{I})$ and H441 cells (J-K) with reconstitution of SLC7A11 and SLC3A2, respectively or in combine following treating with indicated concentration of Dox, in the absence or presence of Fer-1 (1 $\mu \mathrm{M})$ for $24 \mathrm{~h}$. (L-M) SLC7A11 and SLC3A2 were knocked out separately or in combine in H1299 (L) and H441 cells (M) treating with or without indicated concentration of Dox for $24 \mathrm{~h}$, as measured by IB. (N-Q) Cell death (N, P) and lipid ROS generation (O, Q) were measured in $\mathrm{H} 1299$ (N-O) and $\mathrm{H} 441$ cells $(\mathrm{P}-\mathrm{Q})$ with separate or combined KO of SLC7A11 and SLC3A2 following treating with or without indicated concentration of Dox, in the absence or presence of Fer-1 $(1 \mu \mathrm{M})$ for $24 \mathrm{~h}$. Data are presented as means \pm SEMs from three independent experiments. Statistical analysis was performed using one-way ANOVA (B-E, H-K, N-Q). *p < $0.05,{ }^{*} \mathrm{p}<0.01$ and N.S., non-significance. 
A

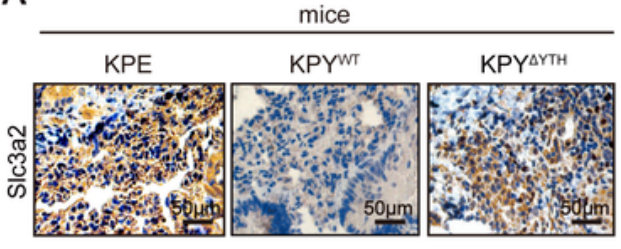

B

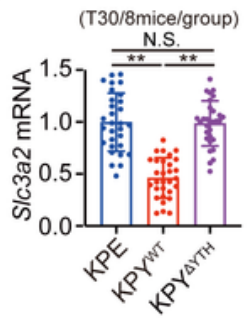

C

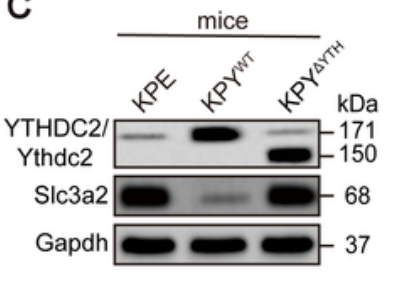

D
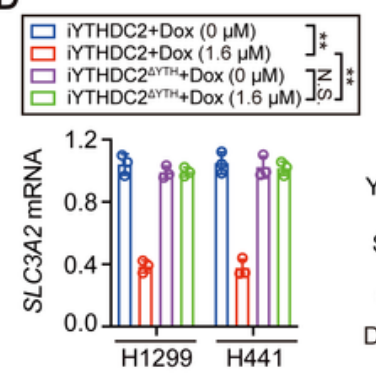

G
E

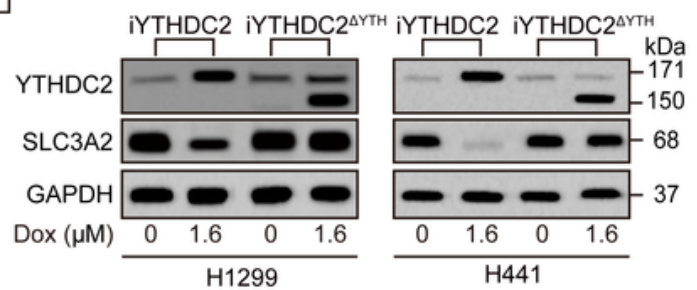

F
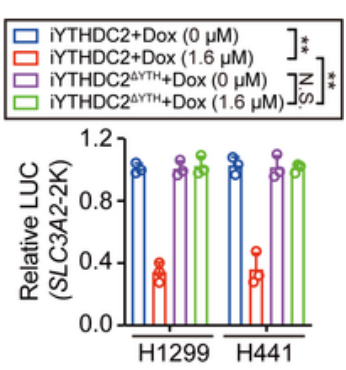

$\mathrm{H}$
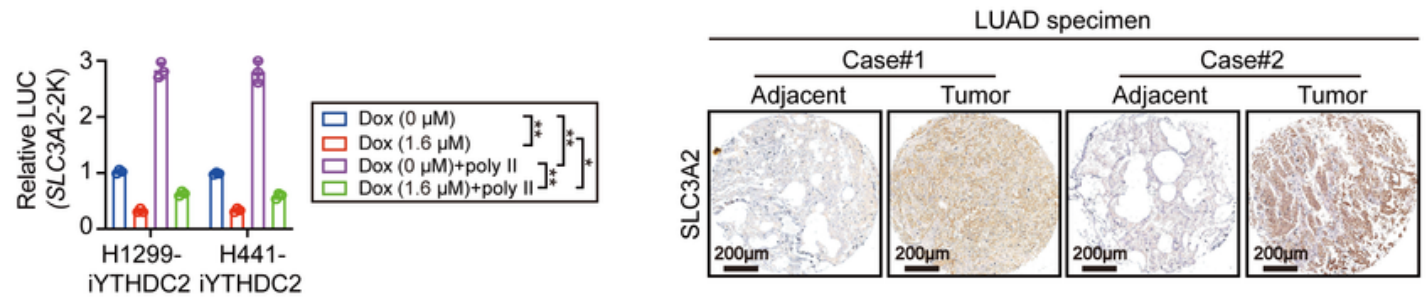

J

LUAD cohort \#1 ( $n=192)$

LUAD cohort \#1 ( $n=192)$

K
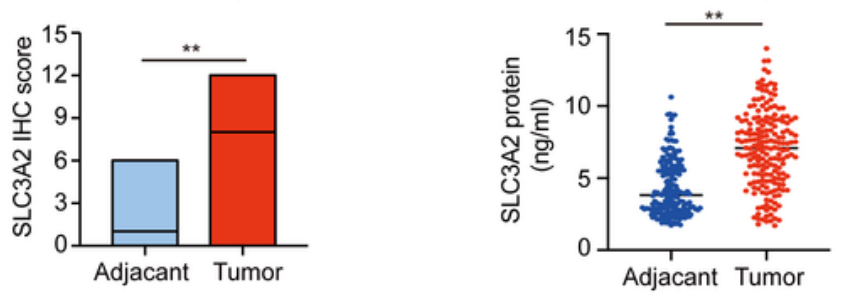

LUAD cohort \#1 ( $n=100)$

L

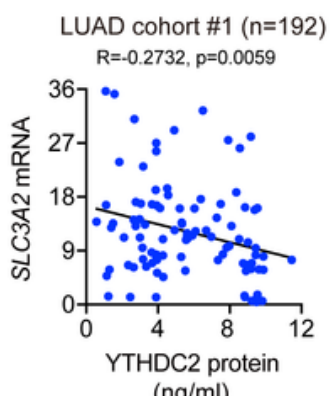

(ng/ml)

M

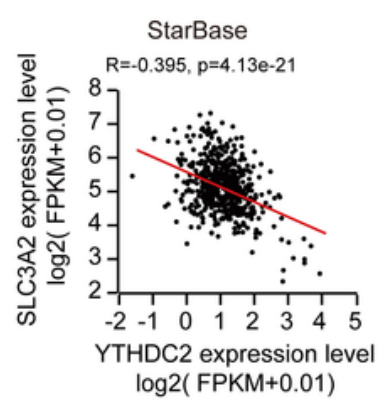

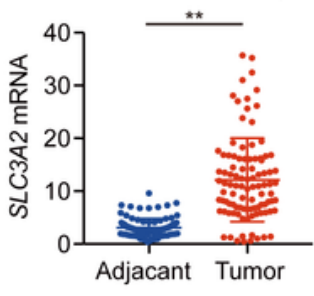

N

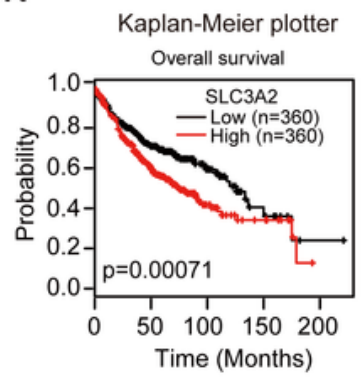

Figure 3

YTHDC2 suppresses SLC3A2 at transcriptional level (A-C) Scl3a2 expression in the LUAD tumors from KPE, KPYWT and KPY $\triangle$ YTH mice, as measured by IHC (A), qPCR (B) and IB (C) ( $n=30$ tumors from 8 mice per group). Scale bar, $50 \mu \mathrm{m}$. (D-E) SLC3A2 expression in Dox-inducible YTHDC2/YTHDC2 $\triangle \mathrm{YTH}$ expressing $\mathrm{H} 1299$ and $\mathrm{H} 441$ cells following treating with or without indicated concentration of Dox for 24h, as measured by qPCR (D) and IB (E). (F) SLC3A2 promoter activity in H1299 and H441 cells 
following induction with or without YTHDC2 or YTHDC2 $\triangle \mathrm{YTH}$ for 24h. (G) SLC3A2 promoter activity in H1299 and H441 cells following induction with or without YTHDC2 for 24h, in the absence or presence of polymerase II. (H-K) SLC3A2 was upregulated in LUAD, as measured by IHC-based TMA (H-I, $n=192)$, $\operatorname{ELISA}(J, n=192)$ and $q P C R(K, n=100)$, Scale bar, $200 \mu \mathrm{m}$. (L) Correlation between YTHDC2 protein and SLC3A2 mRNA in cohort \#1, as analyzed by pearson analysis $(n=192, p=0.0059)$. (M) The correlation between YTHDC2 and SLC3A2 mRNAs revealed by the data from StarBase. $(\mathrm{N})$ The OS between LUAD patients with low or high expression of SLC3A2, as revealed by the data from the Kaplan-Meier plotter database. Data are presented as means \pm SEMs from three independent experiments. Statistical analysis was performed using one-way ANOVA (B, D, F, G), Chi-squared test (I) and t-test (J, K). ${ }^{\star \star} p<0.01$ and N.S., non-significance. 
A

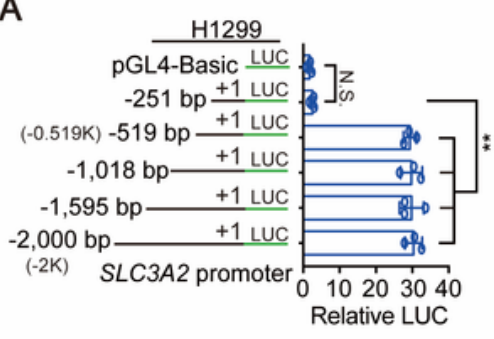

B
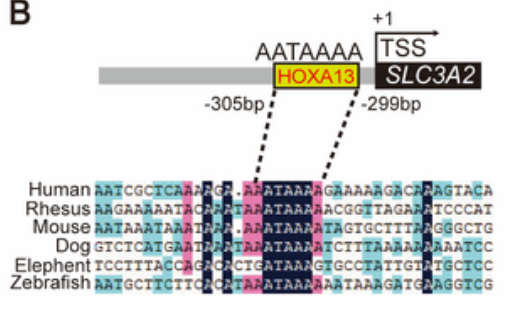

C

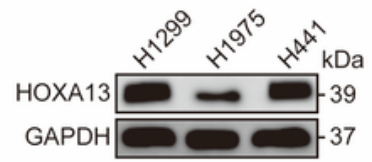

D
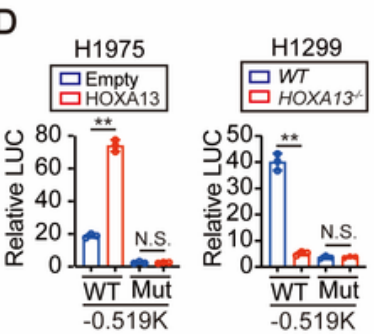

$\mathrm{E}$

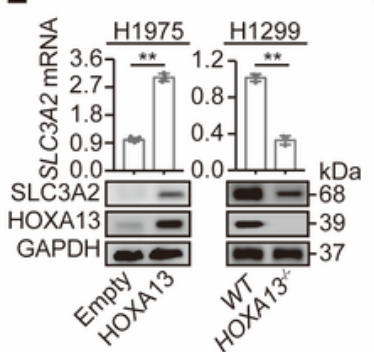

F

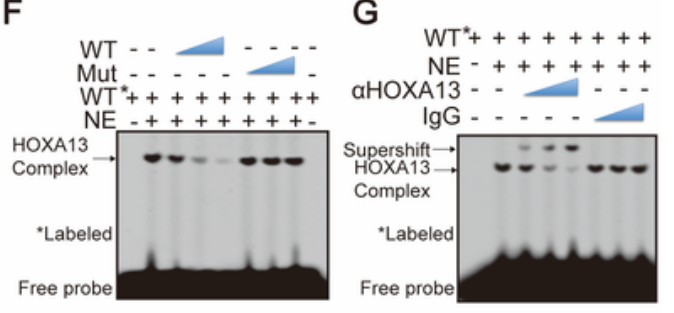

H
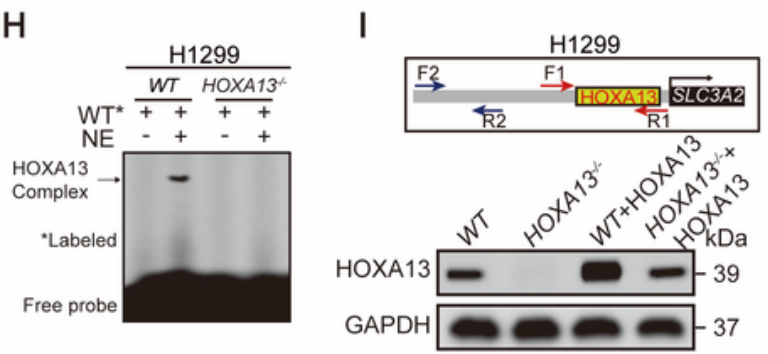

J

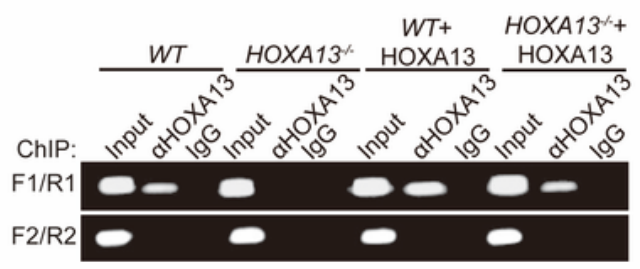

M

N

LUAD cohort \#1 ( $n=192)$
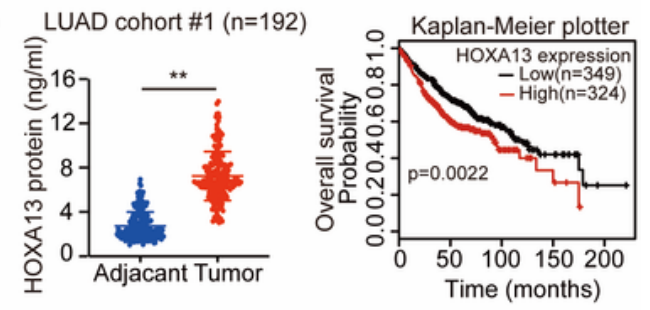

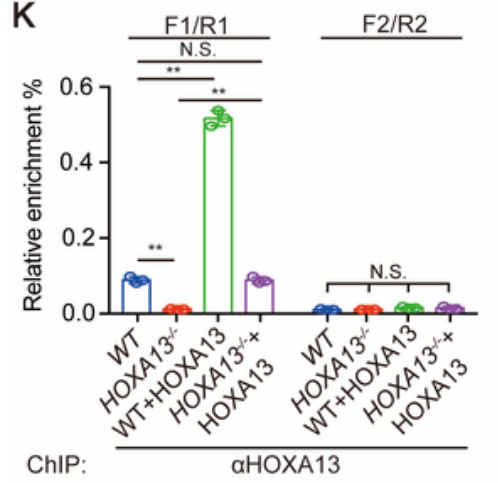

O

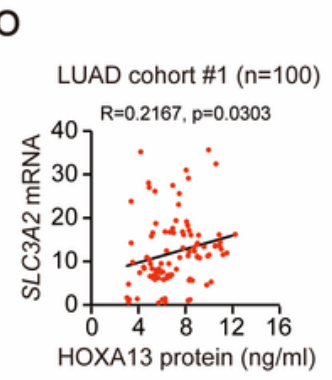

LUAD cohort \#1 $(n=100)$

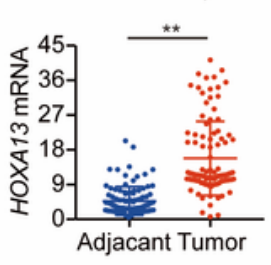

P

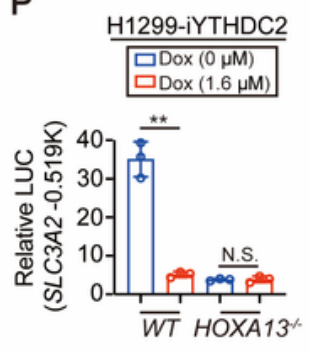

Q

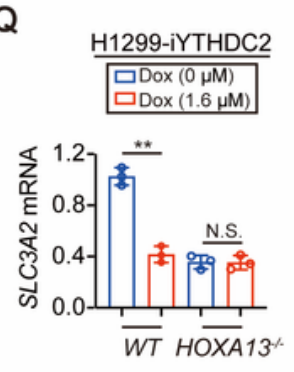

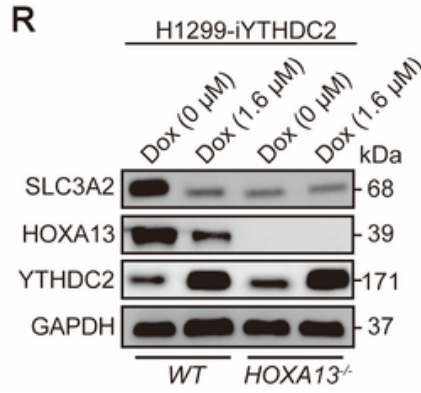

Figure 4

YTHDC2 suppresses SLC3A2 transcription via HOXA13 (A) Luciferase activities from truncated versions of the SLC3A2 promoter. (B) A HOXA13 binding motif of SLC3A2 promoter was conserved among species. (C) The intracellular expression of HOXA13 protein in H1299, H1975 and H441 cells, as measured by IB. (D-E) SLC3A2 promoter with or without intact HOXA13 motif and SLC3A2 expression were measured by Duo-luciferase assay (D), qPCR and IB (E) in H1975 and H1299 cells with HOXA13 
overexpressed or knocked out. (F-H) The interaction between the probes compassing the HOXA13 motif of the SLC3A2 promoter and nuclear exacts (NE) from H1299 cells, as measured by EMSA for different incubation as indicated. (I-K) ChIP was performed using anti-HOXA13 antibodies and control IgG in H1299 cells with or without HOXA13 KO, overexpression or reconstitution. (L-M) HOXA13 expression in tumor and paired adjacent lung tissues in cohort \#1, as measured by $q P C R(L, n=100)$ and ELISA (M, $n=192$ ). (N) OS in LUADs with low or high expression of HOXA13, as revealed by the data from the Kaplan-Meier plotter database. (0) The correlation between SLC3A2 mRNA and H0XA13 protein in cohort \#1, as analyzed by pearson analysis $(p=0.0303, n=100)$. (P-R) YTHDC2 suppressed SLC3A2 promoter activity and expression via HOXA13, as measured by Duo luciferase assay (P), qPCR (Q) and IB (R) in $\mathrm{H} 1299$ cells with or without KO of HOXA13 in the presence or absence of indicated concentration of DoX for $24 \mathrm{~h}$. Data are presented as means \pm SEMs from three independent experiments. Statistical analysis was performed using one-way ANOVA (A, K) and t-test (D, E, L, M, P, Q). **p $<0.01$ and N.S., nonsignificance. 
A

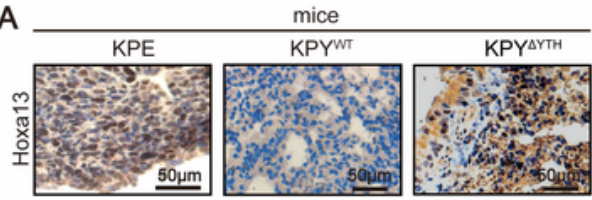

B

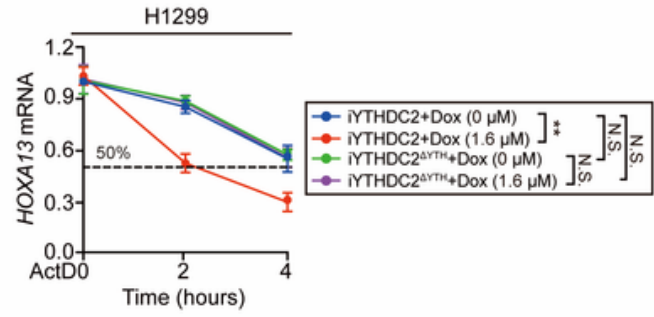

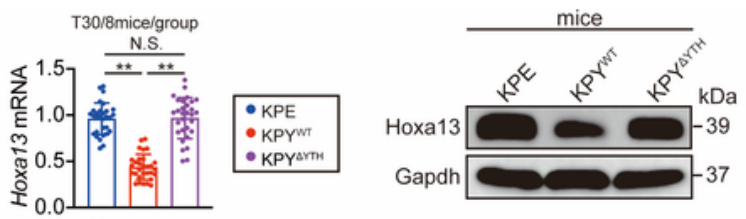

D

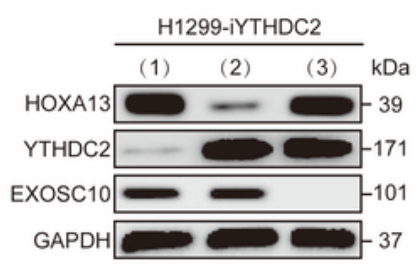

H1975
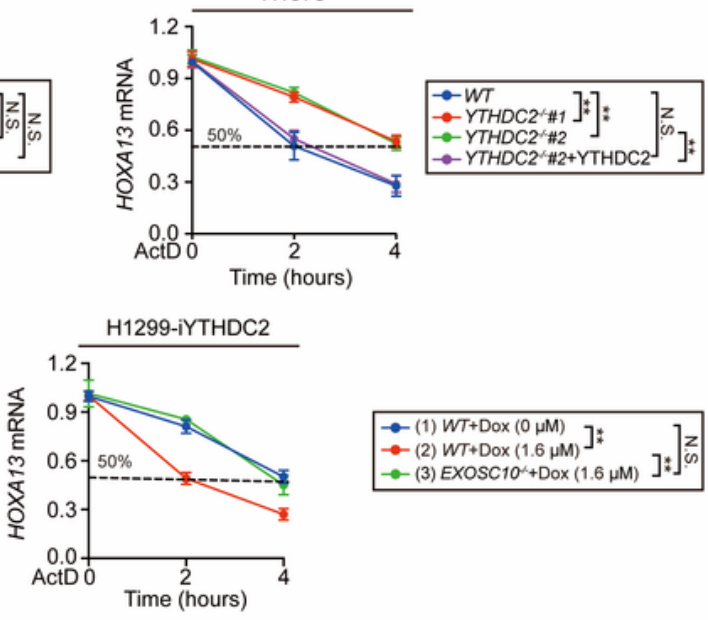

- (1) $W T+\operatorname{Dox}(0 \mu M)$ ]:

$\rightarrow$ (3) EXOSC10 $\left.\left.10^{\circ}+\operatorname{Dox}(1.6 \mu \mathrm{MM})\right]^{*}\right]$ :

E

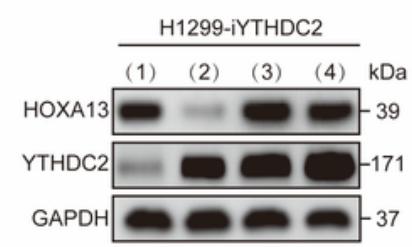

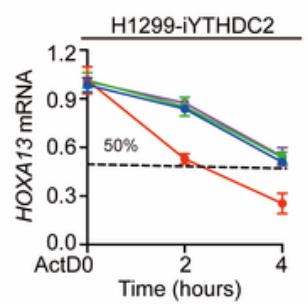

G

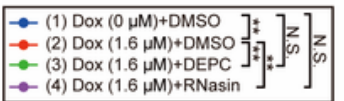

F

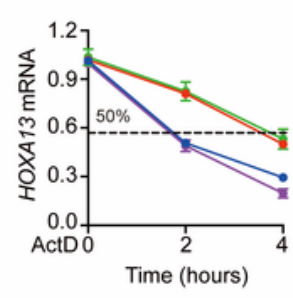

$\mathrm{H}$

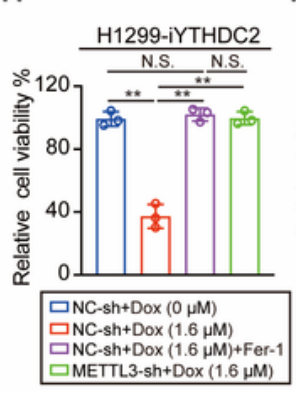

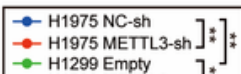

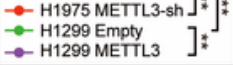

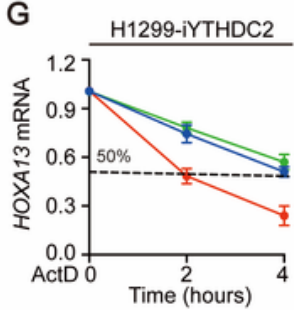

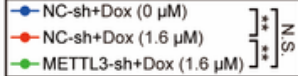

J
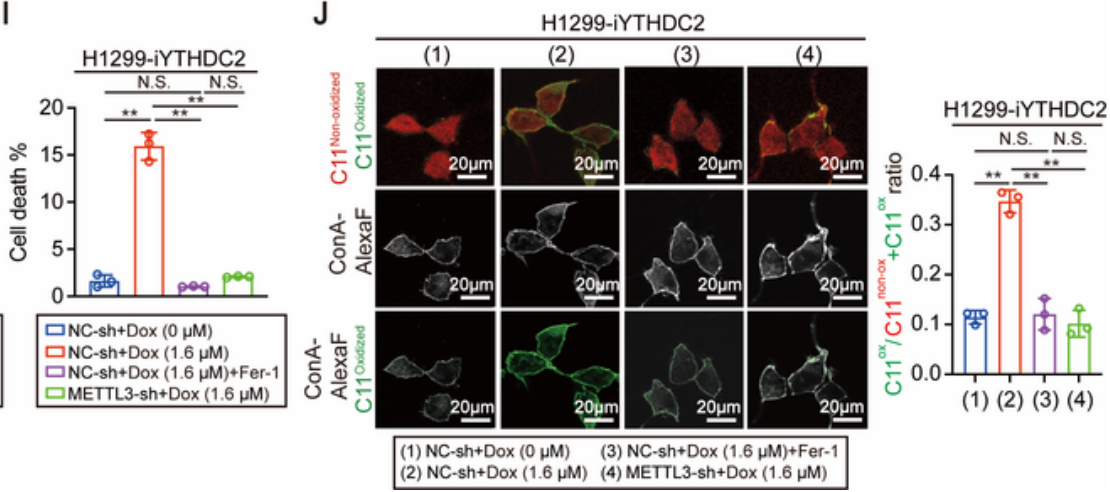

$\begin{array}{ll}\text { (1) NC-sh+Dox }(0 \mu \mathrm{M}) & \text { (3) NC-sh+Dox }(1.6 \mu \mathrm{M})+\mathrm{Fer}-1 \\ \text { (2) NC-sh+Dox }(1.6 \mu \mathrm{M}) & \text { (4) METTL3-sh+Dox }(1.6 \mu \mathrm{M})\end{array}$

Figure 5

YTHDC2 m6A-dependently destabilizes HOXA13 mRNA and induces ferroptosis (A) Hoxa13 expression in the LUAD from KPE, KPYWT and KPY $\triangle Y T H$ mice, as measured by IHC, qPCR and IB. ( $n=30$ tumors from 8 mice per group). Scale bar, $50 \mu \mathrm{m}$. (B-C) ActD chase experiments for HOXA13 mRNA in inducible YTHDC2 or YTHDC2 $\triangle$ YTH-expressing $\mathrm{H} 1299$ cells following treating with or without Dox for 24h (B) and in H1975 cells with or without KO or reconstitution of YTHDC2 (C). (D-E) ActD chase experiments for 
HOXA13 mRNA in inducible YTHDC2-expressing H1299 cells with or without KO of EXOSC10 (D) or in the absence or presence of DEPC or RNasin $(5 \mathrm{U} / \mu \mathrm{L})(\mathrm{E})$ following treating with or without indicated concentration of Dox for 24h. (F) Changes of HOXA13 mRNA stability upon alteration of METTL3. ActD chase experiments were performed for HOXA13 mRNA in H1975 cells with or without METTL3 knockdown or in $\mathrm{H} 1299$ cells with or without METTL3 overexpression. (G) ActD chase experiment for HOXA13 mRNA in inducible YTHDC2-expressing H1299 cells with or without METTL3 knockdown following treating with or without indicated concentration of Dox for $24 \mathrm{~h}$. $(\mathrm{H}-\mathrm{I})$ Cell viability and cell death were measured in inducible YTHDC2-expressing H1299 cells with or without knockdown of METTL3 following treating with or without indicated concentration of Dox in the absence or presence of Fer-1 (1 $\mu \mathrm{M})$ for 24h. (J) Inducible YTHDC2-expressing H1299 cells with or without METTL3 knockdown were probed with C11-BODIPY581/591 for detection of lipid peroxidation and ConA-AlexaF for detection of membrane following treating with or without indicated concentration of Dox in the absence or presence of Fer-1 $(1 \mu \mathrm{M})$ for $24 \mathrm{~h}$. The presence of oxidized C11-BODIPY was also graphed. Scale bar, $20 \mu \mathrm{m}$. Data are presented as means \pm SEMs from three independent experiments. Statistical analysis was performed using one-way ANOVA (A, H-J) and two-way ANOVA (B-G). **p $<0.01$ and N.S., non-significance. 
A

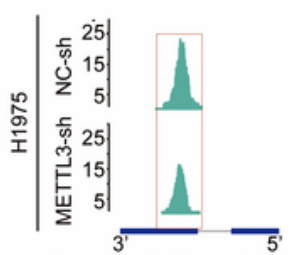

HOXA13 mRNA $\mathrm{m}^{6}$ A peak: $+1140 \mathrm{bp} \sim+1569$ bp (relative to TSS) ... $m^{6} \mathrm{~A}$ site G̈̈AC: $+1517 \mathrm{bp} \sim+1520 \mathrm{bp}$
B
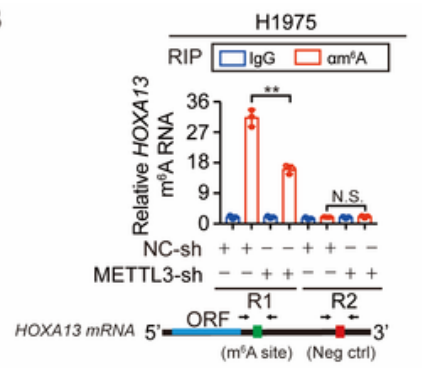

C

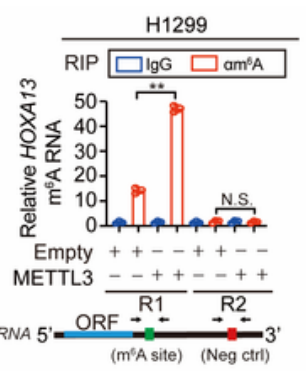

$\mathrm{E}$

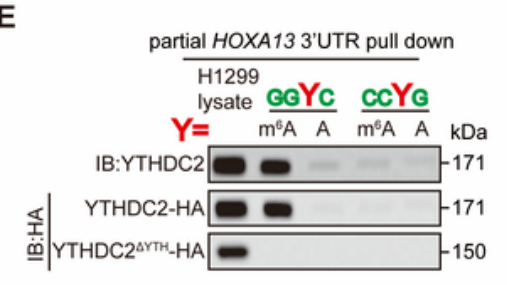

G
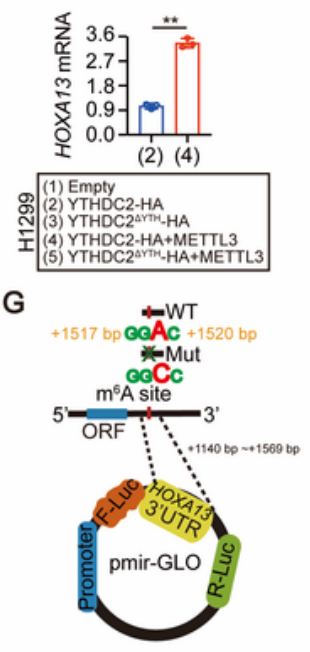
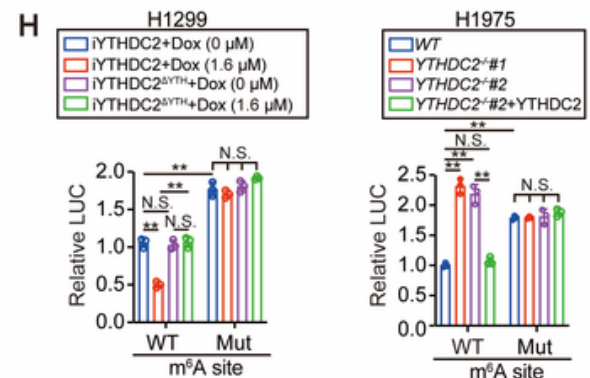

$\mathrm{K}$
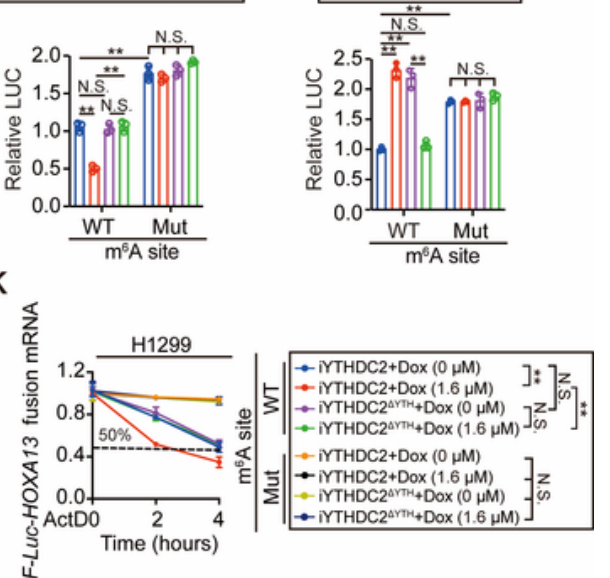

L

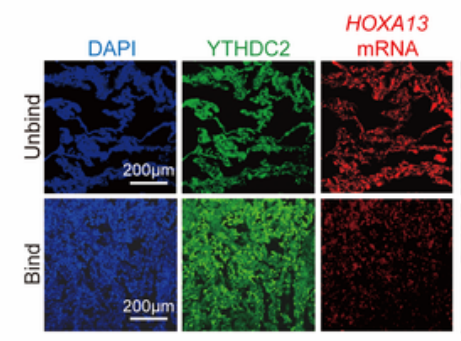

○

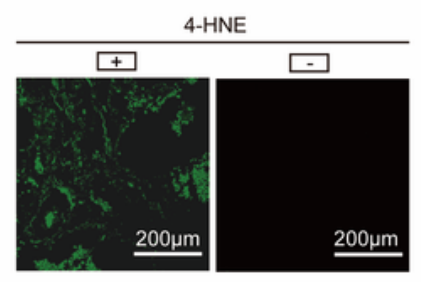

$\mathrm{H} 1975$

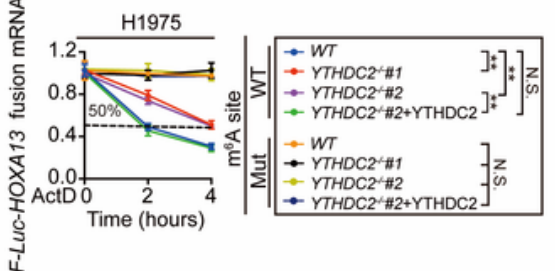

M

N

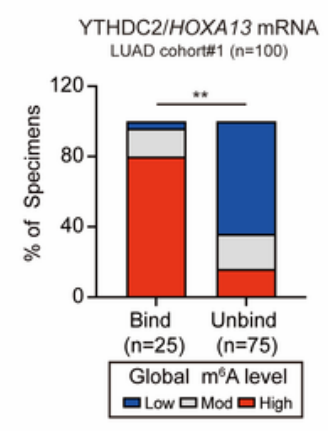

Figure 6

m6A methylation of HOXA13 mRNA is required for YTHDC2 function (A) meRIP-seq for H1975 cells with or without METTL3 knockdown revealed a m6A site within the 3'UTR of HOXA13 mRNA. (B-C) The enrichment of HOXA13 mRNA in RIP experiments performed by using anti-m6A antibodies and control IgG in H1975 cells with or without METTL3 knockdown (B) and in H1299 cells with or without METTL3 overexpression (C). The $\mathrm{R} 1$ region contains the m6A site, while $\mathrm{R} 2$ is an m6A unrelated region. (D) PAR- 
CLIP was performed in HA-tagged YTHDC2- or YTHDC2 $\triangle$ YTH-expressing H1299 cells with or without overexpression of METTL3. The presence of HOXA13 mRNA in the products of PAR-CLIP was also graphed. (E) RNA pull down experiments using the lysates from $\mathrm{H} 1299$ cells and probing by probes containing partial 3'UTR of the HOXA13 mRNA. (F) RIP for HOXA13 mRNA using anti-YTHDC2 antibodies and control IgG in H1975 cells with or without METTL3 knockdown and in H1299 cells with or without METTL3 overexpression. (G) Schematic presentation of the construction for the HOXA13 3'UTR containing pmir-GLO luciferase reporters. $(H)$ Luciferase activity from pmir-GLO reporters containing WT or m6A site-mutated HOXA13 3'UTR in inducible YTHDC2 or YTHDC2 $\triangle$ YTH-expressing H1299 cells following treating with or without indicated concentration of Dox for 24h and in $\mathrm{H} 1975$ cells with KO or reconstitution of YTHDC2. (I) Schematic representation of the primer sets for the detection of WT or m6A site-mutated Firefly luciferase (F-Luc)-HOXA13 fusion mRNA. (J-K) ActD chase experiment for WT or m6A site mutated-F-Luc-HOXA13 fusion mRNA in $\mathrm{H} 1975$ cells with or without KO or reconstitution of YTHDC2 $(\mathrm{J})$ or in inducible YTHDC2 or YTHDC2 $\triangle$ YTH-expressing $\mathrm{H} 1299$ cells following treating with or without indicated concentration of Dox for $24 \mathrm{~h}(\mathrm{~K})$. (L) Representative con-focal images for YTHDC2 and HOXA13 mRNA in human LUAD tissues analyzed using FISH assay. Bind and unbind demonstrate with or without co-localization of YTHDC2 and HOXA13 mRNA. Scale bar, $200 \mu \mathrm{m}$. (M) The frequency for bind and unbind YTHDC2/HOXA13 mRNA phenomenon in human LUAD from cohort \#1. (N) The frequency of different levels of global m6A in LUAD with bind or unbind YTHDC2/HOXA13 mRNA phenomenon in cohort \#1. (0) The representative IF images showing the absence $(-)$ or presence $(+)$ of 4-HNE in human LUAD. Scale bar, $200 \mu \mathrm{m}$. (P) The frequency for the bind or unbind YTHDC2/HOXA13 mRNA phenomenon in cohort \#1 LUADs with absence $(-)$ or presence $(+)$ of 4-HNE. Data are presented as means \pm SEMs from three independent experiments. Statistical analysis was performed using $t$ test (B-D, F), one-way ANOVA $(H)$, two-way ANOVA $(J, K)$ and Chi-squared test $(N, P)$. **p $<0.01$ and N.S., non-significance. 
A

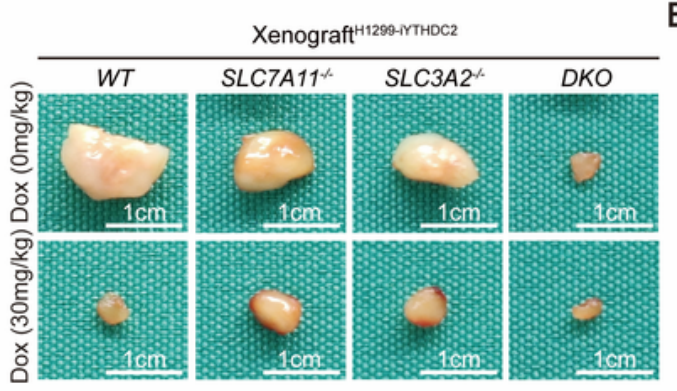

B

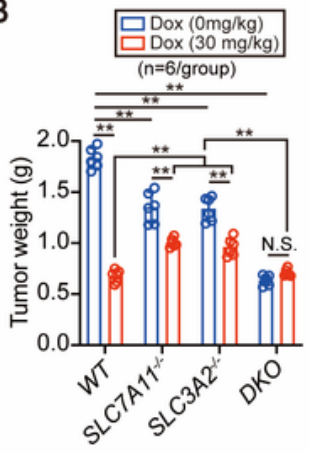

D

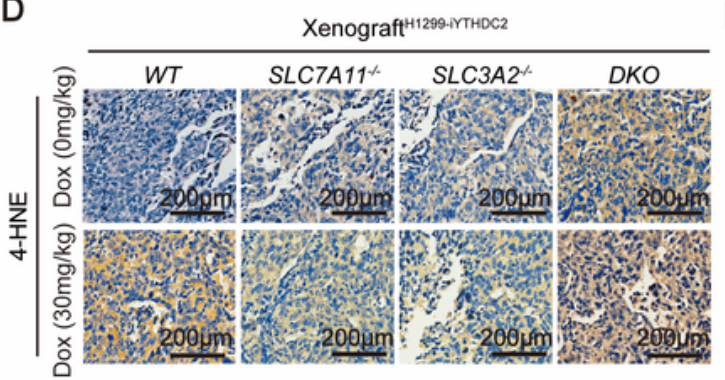

E ${ }^{\text {Dox }(0 \mathrm{mg} / \mathrm{kg})} \mathrm{F}$

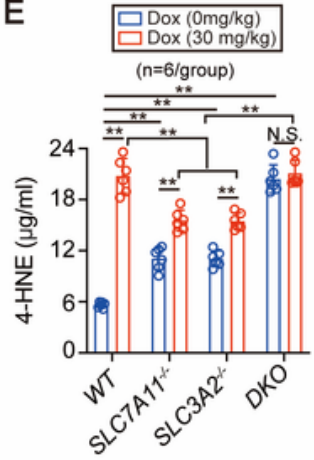

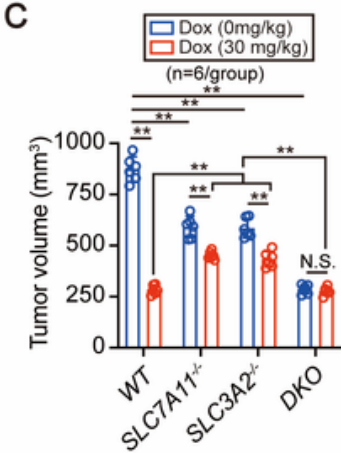

F $\begin{aligned} & \text { Dox ( ( m m } / \mathrm{kgg} \\ & \text { Dox }(30 \mathrm{mg} / \mathrm{kg})\end{aligned}$

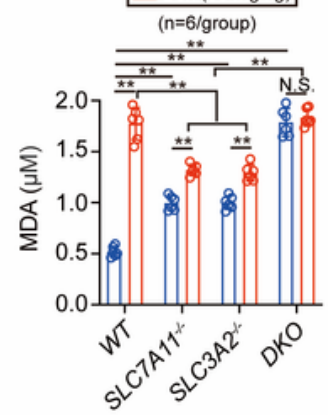

G

$\mathrm{H}$
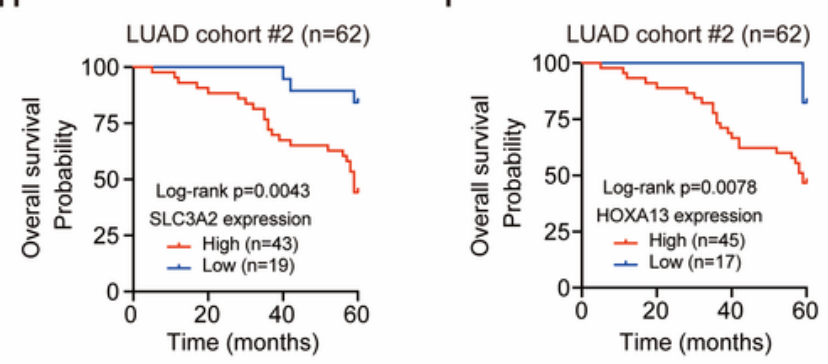

LUAD cohort \#3 ( $\mathrm{n}=30$ )

J

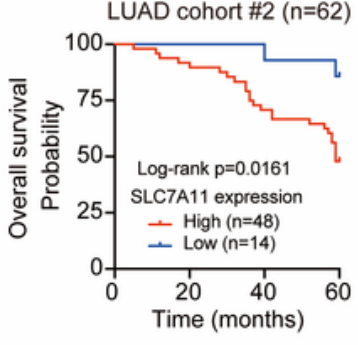

**
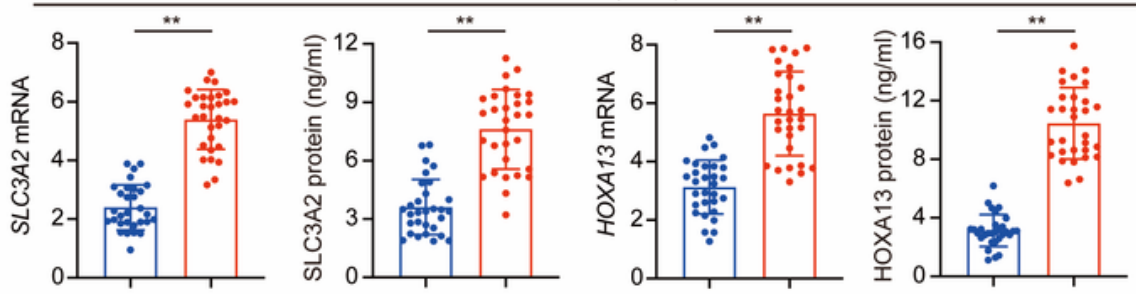

- Adjacent

- Tumor

K

LUAD cohort \#4 ( $n=20 /$ group)
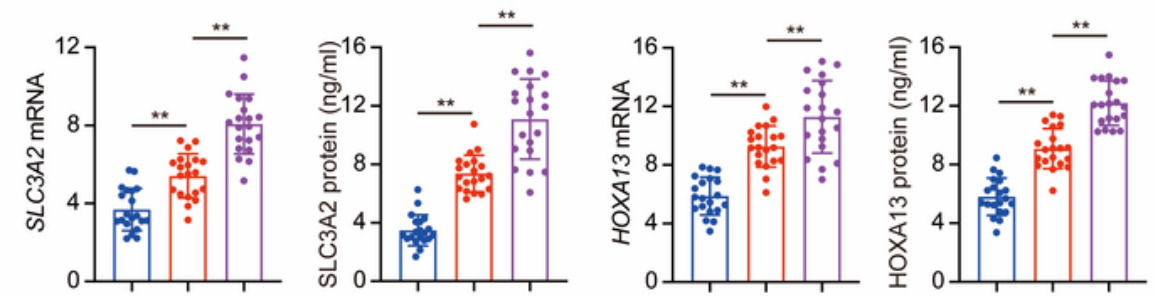

- Stage I
- Stage II
- Stage III

Figure 7

The potential usage of YTHDC2 to impair tumor growth and further clinical significance of this study (AC) Tumor burden in xenograft mouse models inoculated with inducible YTHDC2-expressing H1299 cells with or without separate or combine KO of SLC7A11 and SLC3A2 following administrating with or without indicated concentration of Dox for another 14 days. Representative images of tumor are shown (A), and Tumor weight (B) and volume (C) are also graphed. Scale bar, $1 \mathrm{~cm}$. (D-F) Lipid peroxidation in 
the tumors same as those in panel (A-C) were indicated by measuring 4-HNE (D-E) and MDA (F). Scale bar, $200 \mu \mathrm{m}$. (G-I) OS of YTHDC2-suppressed acinar LUADs in cohort \#2 with high or low expression of SLC7A11 ( $G, p=0.0161)$, SLC3A2 ( $H, p=0.0043)$ and HOXA13 $(l, p=0.0078)$. $(J)$ SLC3A2 and H0XA13 expression in tumor and its paired adjacent lung tissues in cohort \#3, as measured by qPCR and ELISA. (K) SLC3A2 and HOXA13 expression in LUAD from cohort \#4 with indicated stage. Data are presented as means \pm SEMs from three independent experiments. Statistical analysis was performed using one-way ANOVA (B, C, E, F, K), log-rank test (G-I) and $t$ test (J). **p $<0.01$ and N.S., non-significance.

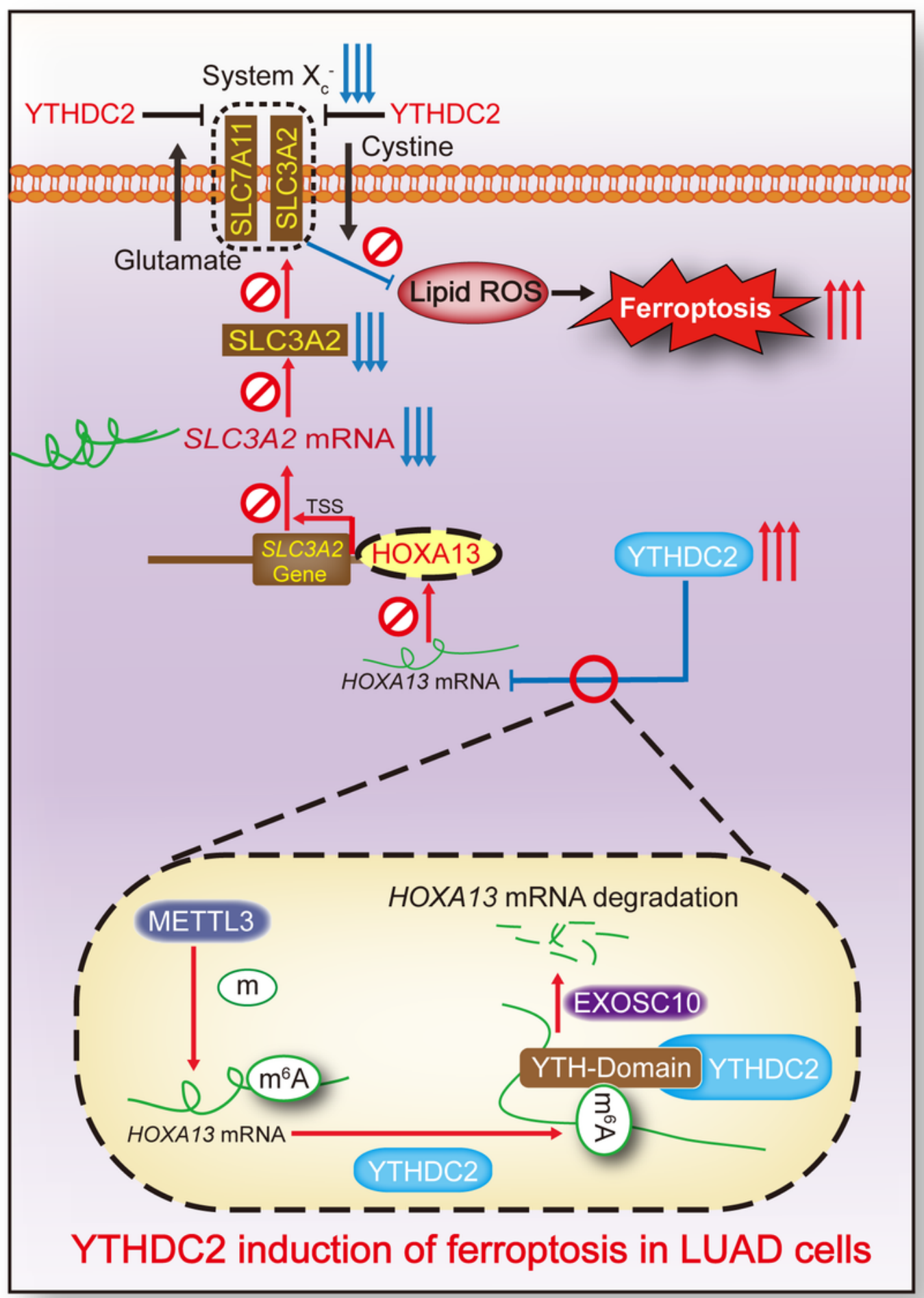


Figure 8

Schematic presentation of the study Briefly, the m6A reader YTHDC2 is an endogenous ferroptosis inducer and its function is to suppress system XC-. In addition to what we've previously reported that the mRNA stability of SLC7A11 is suppressed by YTHDC2, in the present study we also found that SLC3A2, another subunit of system XC- can also be suppressed by YTHDC2 via targeting HOXA13-mediated transcription of SLC3A2, and the m6A methylation and subsequent destabilization of HOXA13 mRNA is a prerequisite for this process.

\section{Supplementary Files}

This is a list of supplementary files associated with this preprint. Click to download.

- Supplementary.docx 